\title{
Inventories and Endogenous Stackelberg Leadership in Two-period Cournot Oligopoly
}

\author{
Sébastien Mitraille* \\ Toulouse Business School
}

\author{
Michel Moreaux \\ Toulouse School of Economics (IDEI and LERNA)
}

July 27,2011

\begin{abstract}
Two-period Cournot competition between $n$ identical firms producing at constant marginal cost and able to store before selling has pure strategy Nashperfect equilibria, in which some firms store to exert endogenously a leadership over rivals. The number of firms storing balances market share gains, obtained by accumulating early the output, with losses in margin resulting from increased competition and higher operation costs. This number and the industry inventories are non monotonic in $n$. Concentration (HHI) and competition increase due to the strategic use of inventories.
\end{abstract}

JEL Classification: L13, D43

Keywords: Inventories, Cournot, Oligopoly, Endogenous Leadership.

Acknowledgements: Thanks to Eric Maskin and to participants of the Summer School on Game Theory and its Applications (Free University of BozenBolzano), to Gianni De Fraja, Ludovic Renou, to seminar participants at the University of Leicester and at the University of Montpellier I (LAMETA), and to Joseph Harrington for their comments on an earlier version. Thanks also to participants of the IIOC (Savannah), CEA (Montreal), ESEM (Vienna) and ASSET (Lisbon) conferences.

${ }^{*}$ Corresponding author. Toulouse Business School. 20 Bd Lascrosses - BP7010 - 31068 Toulouse Cedex 7 - France. Phone: +33 (0) 5612948 44. Fax: +33 (0) 5612949 94. E-mail: s.mitraille@esc-toulouse.fr 


\section{Introduction}

Inventory management is a key issue for many firms, be they large or small: wellknown management theories emphasize the need to stay lean, the gain to proceed with just-in-time deliveries and zero inventories, or on the contrary the possibility to benefit from economies of scale by purchasing or producing the economic-orderquantity (Arrow, Harris and Marschak [1951], Zipkin [2000]), and hence storing. The importance of inventories in economic analysis has also been recognized. For instance Arvan and Moses [1982] show that economies of scale in the production of a storable good lead a monopolist to adjust its output in the long run. Social losses then differ compared to the static case. Blanchard [1983] investigates the behaviour of finished good inventories in the U.S. automobile industry ${ }^{1}$. Descriptive statistics show that "production smoothing is not the dominant element of inventory behavior and that target inventory is probably a function of current sales" (p.374, our emphasis), and econometric estimations confirm this finding. Using a sample of U.S. firms from the good-producing manufacturing industries, Amihud and Mendelson [1989] show that firms hold larger level of inventories the greater their market power. However an interesting theoretical question is to understand how a target level of inventories is determined when firms are part of an oligopoly, as for example the U.S. automobile industry, and more broadly to investigate how inventory levels vary with the degree of competition. Is it possible to revert the causal relationship and find that larger equilibrium level of inventories allow firms to enjoy larger equilibrium market shares and consequently greater market power on an oligopolistic market? Moreover how is the equilibrium level of inventories affected by changes in the degree of competition? Answering these questions is the object of this study.

Under constant returns-to-scale and in the absence of any capacity constraint or uncertainty, we show that Cournot oligopolistic competition in which $n$ firms produce to store in a first period, before producing again and selling on the market in second period, has a Nash-perfect equilibrium in pure strategy no matter the number of firms in competition or the cost of storage. Equilibria can be multiple and differ according to the number of firms which store. The static Cournot-Nash

\footnotetext{
${ }^{1}$ Blanchard uses monthly data from January 1966 to December 1979 for 10 divisions of the main U.S. manufacturers: 5 from GM, 2 from Ford, 2 from Chrysler and 1 from American Motors.
} 
outcome where no firm stores is the expected unique equilibrium of the game when the cost of storage is large enough, but outside this range it is not an equilibrium anymore. For intermediate or small values of the cost of storage, equilibria are such that a subset of firms is storing a positive quantity to exert endogenously some Stackelberg leadership over the rest of firms who behave as followers, who do not store and who produce and sell in second period only. The number of firms storing at equilibrium results from the trade-off between a larger market share, gained thanks to the endogenous commitment to be more aggressive on the market by accumulating early the output, and a smaller margin, due to the increase in competition among firms and to the increase in the costs of operations that include now a cost of storage. The profitability of the strategy that consists in increasing inventories to credibly commit to gain market shares is therefore larger when a few firms are in competition on the market, and decreases when $n$ increases, which suggests a non monotonicity of the equilibrium in the number of competitors. We confirm this intuition and show that the number of firms storing and the aggregate inventories at equilibrium can be non monotonic with the number of firms in competition. Finally as the equilibria are asymmetric,- except of course the Cournot one when it occurs -, concentration increases compared to what it would be at the static Cournot-Nash equilibrium, with identical demand and costs. We show that the Herfindhal-Hirschman concentration index (HHI) is strictly larger than the value it obtains in static Cournot competition, while the price-cost margin is strictly lower due to the increase in competition caused by inventories.

We illustrate these findings and study the effect of an entry of competitors on the set of equilibria with an example. The number of firms storing and the aggregate inventories are non monotonic with respect to the number of firms in competition. As predicted, the HHI and the aggregate sales both reach levels strictly larger than what they do in static Cournot competition when storing to gain market shares is a profitable strategy: the market is more concentrated and more competitive than what it would be if storage were impossible. Together with our theoretical findings, these results suggest that observing a mass of firms storing is more likely on oligopolistic markets than on market structures closer to the duopoly or to perfect competition. Moreover they also suggest that the HHI cannot be used as the sole measure of competitiveness on markets where the finished product is storable and 
storage is used to exert some market share leadership.

The commitment value of inventories in two-period Cournot duopolistic competition has been recognized in the literature since the path-breaking articles of Arvan [1985] and Ware [1985]. Both articles identify that once the costs of production have been sunk, inventories endow firms with a capacity from which they can sell while suffering no marginal cost of production. This creates an endogenous discontinuity in the marginal cost the firm is facing when deciding how much to produce and sell on the market, which may result in a first mover advantage. This effect, obviously linked to Dixit [1980], implies the existence of asymmetric equilibria even if firms are identical with respect to their production technology, and produce and sell simultaneously in every period ${ }^{2}$. In an independent series of papers, Saloner [1987] and Pal ([1991], [1996]) study duopolistic Cournot competition with advance production and constant returns-to-scale, to show that multiple equilibria may arise, but that this multiplicity may disappear with cost variations ${ }^{3}$. We generalize these results to the case of an oligopoly and we study the effect of entry. The resolution of this game is made by constructing the backward reaction mapping proposed by Novshek [1984] (see also Bamon and Frayssé [1985]), that is by constructing the best reply of each firm to the aggregate sales of the industry compatible with this firm optimization program. Due to the use of inventories, backward reaction mappings turn out to be different across firms even if firms are ex-ante identical, but for each distribution of inventories these backward reaction mappings determine a unique and possibly asymmetric Nash equilibrium in pure strategy to the second period Cournot sub-game.

Our results are closely connected to the broader and more recent literature studying the industrial organization of commodity markets ${ }^{4}$ and the strategic role of forward markets. In their seminal article, Allaz and Vila [1993] show that forward markets may be used by Cournot duopolists to compete for the Stackelberg leadership. The equilibrium of the game is however symmetric, firms competing in advance

\footnotetext{
${ }^{2}$ See Ware [1984] for a different version of the game in Dixit [1980].

${ }^{3}$ Romano and Yildirim (2005) partly generalize the result of Saloner to the broader class of duopolistic games of capital accumulation. Contrary to Saloner they focus on games in which the Stackelberg equilibrium in unique.

${ }^{4}$ See Anderson [1984] for earlier contributions.
} 
for a larger share of the market ${ }^{5}$. Our paper differs from these studies in the type of commitment considered: while forward sales can be re-interpreted as a commitment which places the entire industry in front of a reduced residual demand when forward contracts are exerted, inventories are committing only the firm which is holding them, source of the asymmetry of the Nash equilibrium. The use of inventories and forward trading in imperfect competition has also been studied in the context of longer dynamic with uncertain demand and costs (see Kirman and Sobel [1974] and Thille ([2003], [2006])).

The remaining of the paper is organized as follows: section 2 presents the model; section 3 shows that for any distribution of ordered inventories across firms, it exists a unique sub-game equilibrium in which market shares can be asymmetric. Then section 4 derives the equilibria of the game and study the effect of entry. Section 5 summarizes our results and concludes.

\section{The Model}

We consider an homogenous market with $n$ Cournot competitors indexed by $i, i \in$ $I=\{1, \ldots, n\}, n \geq 2$, competing over two periods indexed by $t=1,2$. Let $q_{t}^{i}$ be the production level of firm $i$ in period $t, q_{t}=\left(q_{t}^{1}, \ldots, q_{t}^{n}\right)$ the production vector in period $t, Q_{t}=\sum_{i} q_{t}^{i}$ the aggregate output and $Q_{t}^{-i}=\sum_{j \neq i} q_{t}^{j}$ the aggregate output of firms $j \neq i$.

Production may be undertaken in any period, but the market opens in period 2 only. Let $s^{i}$ be the quantity sold by firm $i$ in period 2. Individual sales cannot be larger than the total output available in period $2, s^{i} \leq q_{1}^{i}+q_{2}^{i}$. Finally we denote $s=\left(s^{1}, \ldots, s^{n}\right)$ the sales vector, $S=\sum_{i} s^{i}$ the aggregate sales level and $S^{-i}=\sum_{j \neq i} s^{j}$ the aggregate sales of firm $j \neq i$.

Firms have access to the same constant returns production technology and the same factor prices. Each one is a "small" buyer in the factor market, taking prices as given. Hence all firms have the same constant marginal cost of production denoted by $c, c>0$.

We assume that pure inventory costs are nil, excepted the opportunity cost of

\footnotetext{
${ }^{5}$ See Thille and Slade [2000], Mahenc and Salanié [2004], and Liski and Montero [2006] for different specifications which modify Allaz and Vila's result.
} 
working capital. Assuming that the capital market is perfectly competitive, and denoting by $\rho$ the interest rate, the only opportunity cost is the cost of producing in period 1 rather than in period 2, that is $\rho c q_{1}^{i}$ in terms of value in period 2. Under a free disposal assumption insuring that inventories unsold at the end of period 2 can be disposed off at zero cost, the total cost of any production and sale plan is given by (C.1), in period 2 value:

Assumption (C.1) For any firm $i \in I$ and any plan $\left\{\left(q_{1}^{i}, q_{2}^{i}, s^{i}\right): s^{i} \leq q_{1}^{i}+q_{2}^{i}\right\}$, the total cost incurred in second period, $C_{2}^{i}\left(q_{1}^{i}, q_{2}^{i}, s^{i}\right)$, is given by:

$$
C_{2}^{i}\left(q_{1}^{i}, q_{2}^{i}, s^{i}\right)=(1+\rho) c q_{1}^{i}+c q_{2}^{i}
$$

Introducing an interest rate allows us to introduce cost differentials between periods that help to select the number of equilibria ${ }^{6}$; we discuss more broadly this issue after having stated our main results in section 4 .

The market demand function is assumed to be linear. This insures that the Stackelberg equilibrium is unique, a useful but not crucial property of the payoff functions $^{7}$. Without loss of generality we assume that its slope is equal to -1 . Thus:

Assumption (D.1) Let $P(S)$ be the inverse demand function, then

$$
P(S)=\max \{a-S, 0\}, \quad a>0 .
$$

For the ease of the analysis most of the discussion is lead under the following assumption:

Assumption (A.1) The intercept of the inverse demand $a$ and the marginal cost of production c satisfy

$$
c \leq a \leq 3 c .
$$

\footnotetext{
${ }^{6}$ This relates to Pal (1991)'s extension of the work of Saloner (1987).

${ }^{7}$ Neither Arvan (1985) nor Saloner (1987) results for a duopoly rely on this assumption. Assuming a concave inverse demand to work under a more general quasi-concavity assumption on the payoff function as in Romano and Yildirim (2005) would be a costly alternative in terms of mathematical content. We chose to assume quadratic payoffs to state our results in the simplest form possible.
} 
We discuss the importance of this assumption in section 4; it basically guarantees that the textbook Stackelberg equilibrium is a feasible outcome of our game. If it is relaxed the asymmetric equilibrium still exists in pure strategies but the leader output is slightly smaller than the textbook Stackelberg output. This feature does not occur in capital accumulation games ${ }^{8}$ as players cannot reduce their commitment, while on the contrary in our game players are allowed to sell less than their inventories. Moreover we adopt the convention that if firms inventories differ, then firm 1 is the one with the highest level of inventories, firm 2 is the one with the second highest level of inventories, ..., and so on.

Convention: Firms are indexed by decreasing order of period 1 production levels,

$$
q_{1}^{1} \geq q_{1}^{2} \geq \ldots \geq q_{1}^{n-1} \geq q_{1}^{n}
$$

We assume that any firm can observe all the period 1 production levels but cannot observe period 2 production and sale levels of its competitors. We discuss this assumption after having presented firms payoffs at the end of this section. Under this assumption the strategy of firm $i$, denoted by $\sigma^{i}$, is a 3-tuple:

$$
\sigma^{i}=\left\{q_{1}^{i}, \widetilde{q}_{2}^{i}, \widetilde{s}^{i}\right\}
$$

where:

$$
q_{1}^{i} \in \mathbb{R}_{+}, \quad \widetilde{q}_{2}^{i}: \mathbb{R}_{+}^{n} \rightarrow \mathbb{R}_{+}, \quad \widetilde{s}^{i}: \mathbb{R}_{+}^{n+1} \rightarrow \mathbb{R}_{+}
$$

with $\widetilde{s}^{i}$ satisfying the following condition:

$$
\forall\left(q_{1}, q_{2}^{i}\right) \in \mathbb{R}_{+}^{n+1}, \widetilde{s}^{i}\left(q_{1}, q_{2}^{i}\right) \leq q_{1}^{i}+q_{2}^{i}
$$

To any n-tuple of strategy $\sigma, \sigma=\left\{\sigma^{1}, \ldots, \sigma^{i}, \ldots, \sigma^{n}\right\}$, corresponds a payoff function $\pi^{i}$ for firm $i$ given by, in period 2 value:

$$
\pi^{i}\left(\sigma^{i}, \sigma^{-i}\right)=P\left(\sum_{j \neq i} \widetilde{s}^{j}\left(q_{1}, \widetilde{q}_{2}^{j}\right)+\widetilde{s}^{i}\left(q_{1}, \widetilde{q}_{2}^{i}\right)\right) \widetilde{s}^{i}\left(q_{1}, \widetilde{q}_{2}^{i}\right)-C_{2}^{i}\left(q_{1}^{i}, \widetilde{q}_{2}^{i}, \widetilde{s}^{i}\left(q_{1}, \widetilde{q}_{2}^{i}\right)\right) .
$$

We are searching for the sub-game perfect Nash equilibrium in pure strategies of this game. The possibility of finding mixed strategy Nash equilibria is discussed in

${ }^{8}$ See Saloner (1987) or Romano and Yildirim (2005). 
section 4 (see Pal (1996)).

Before analyzing our game, let us discuss the impact of the non-observability of first period actions on our results. Doing a parallel with the way non-observability modifies the impact of an exogenous commitment ${ }^{9}$, we conjecture that the nonobservability of inventories would not jeopardize our results provided that we introduce a communication technology precise enough. As Schelling (1960) pointed out, the player which has a commitment must make sure the other players know this commitment: it needs a communication technology precise enough. In our paper signals could for example be randomly drawn on inventory levels higher when inventories are large than when they are small. This assumption seems realistic in practice: for example industry reports, mid-year financial statements, or sales employees bargaining with customers may be seen as signals observed by competitors from which posterior beliefs can be derived. We leave the study of this possibility for another paper.

\section{Sub-game equilibria}

We describe now the sub-game equilibria for each vector of inventories chosen in period $1, q_{1}$ : proposition 1 presents the aggregate oligopolistic sales at the Nash equilibrium of each sub-game. To each aggregate sales corresponds a unique vector of equilibrium individual sales. As expected, individual and aggregate sales in equilibrium depend on the level of inventories produced by firms in period 1, and differ depending on how large individual inventories are. The conditions on inventories characterizing each sub-game can be expressed as differences between the marginal revenues obtained by firms when selling their inventories and the effective marginal costs of production. The formal proof of these results is given in appendix A. We now explain the main steps leading to proposition 1.

The effective marginal cost of production of Cournot oligopolists storing their finished product is linked to, but differ from, the marginal cost of production $c$ as follows. As the cost of production of inventories is sunk when selling on the market in second period, inventories can be thought of as an exhaustible capacity from

\footnotetext{
${ }^{9}$ See Bagwell [1991], Van Damme and Hurkens [1997] and Maggi [1998].
} 
which firms can sell without producing as long as the quantity currently sold is smaller than the quantity previously stored. The marginal cost of supplying a unit from inventories is therefore equal to 0 , as long as inventories are not exhausted. As soon as its sales exceed its inventories, a firm must produce again and suffers a positive marginal cost of production. Consequently bringing inventories of the finished product from one period to another modifies the economic behaviour of a firm by modifying its second period competitive supply. Although this effect of inventories has already been explained ${ }^{10}$, and obviously relates to Dixit [1980], it is useful to present it differently from older studies by introducing formally the effective marginal cost of production of each firm, $\gamma^{i}\left(s^{i}, q_{1}^{i}\right)$, given by

$$
\gamma^{i}\left(s^{i}, q_{1}^{i}\right)= \begin{cases}0 & \text { if } s^{i} \leq q_{1}^{i} \\ c & \text { if } s^{i}>q_{1}^{i}\end{cases}
$$

Even if technologies are ex-ante identical, effective marginal costs differ across firms once inventories have been produced, and present a firm-specific jump at $s^{i}=q_{1}^{i}$. The set of economically feasible sales at a given market price is therefore larger when firms store their output than when they do not: firms are now ready to sell up to their inventories $q_{1}^{i}$ if the market price $p$ goes below their marginal cost of production $c$. Consequently, by storing a quantity $q_{1}^{i}$ large enough, a Cournot competitor may resist to an increase in its opponent sales by still preferring to release its inventories $q_{1}^{i}$ on the market rather than reducing its sales. This effect of inventories can be derived by confronting $\gamma^{i}\left(s^{i}, q_{1}^{i}\right)$ to the marginal revenue $m^{i}\left(s^{i}, S^{-i}\right)$,

$$
m^{i}\left(s^{i}, S^{-i}\right)=a-2 s^{i}-S^{-i}
$$

to obtain the best response of a firm to an increase in sales of its competitors. Given the form of the effective marginal cost of production $\gamma^{i}$, the individual best reply shows the three different types of behaviour of firm $i$, depending on competitors sales $S^{-i}$ and on initial inventories $q_{1}^{i}$. If given $q_{1}^{i}$, competitors sales $S^{-i}$ are such that the marginal revenue to sell $q_{1}^{i}, m^{i}\left(q_{1}^{i}, S^{-i}\right)$, exceeds the marginal cost $c$, then firm $i$ produces again in second period and sells more than $q_{1}^{i}$. If $m^{i}\left(q_{1}^{i}, S^{-i}\right)$ is lower than the marginal cost $c$ but positive, then firm $i$ sells exactly its inventories. Finally if $m^{i}\left(q_{1}^{i}, S^{-i}\right)$ is strictly negative, then firm $i$ is better off selling less than its

\footnotetext{
${ }^{10}$ See Arvan [1985] and Ware [1985]
} 
inventories. Individual best responses are given by

$$
\widehat{s}^{i}\left(S^{-i}, q_{1}^{i}\right)= \begin{cases}\frac{1}{2}\left(a-c-S^{-i}\right) & \text { if } S^{-i} \leq a-c-2 q_{1}^{i} \\ q_{1}^{i} & \text { if } S^{-i} \in\left[a-c-2 q_{1}^{i}, a-2 q_{1}^{i}\right] \\ \frac{1}{2}\left(a-S^{-i}\right) & \text { if } a-2 q_{1}^{i} \leq S^{-i}\end{cases}
$$

Inventories therefore create firm-specific kinks in firms best replies, the firm best reply becomes locally inelastic to an increase in its competitors sales, and finally for any given level of sales of its competitors, the level of output a firm sells is higher when it owns inventories than when it does not. This is the source of the asymmetry of the Nash equilibrium in our game.

To find all the equilibria of the game, we start to define the aggregate sales $S$ compatible with the maximisation program of an individual firm $i$, by adding $S^{-i}$ to the best response $\widehat{s}^{i}\left(S^{-i}, q_{1}^{i}\right)$. We denote this function $S_{i}^{\bullet}\left(S^{-i}, q_{1}^{i}\right)$, and we invert it to find aggregate sales of the competitors of firm $i$ compatible with firm $i$ maximisation program; we denote $S^{-i}\left(S, q_{1}^{i}\right)$ this function. Finally, we deduce the individual sales of firm $i$ which best respond to aggregate sales $S$ and which are compatible with firm $i$ 's optimization, by solving $S-s^{i}=S^{-i}\left(S, q_{1}^{i}\right)$ with respect to $s^{i}$. This last operation gives us the backward reaction mapping $s^{i}\left(S, q_{1}^{i}\right)$. We then aggregate all the backward reaction mappings, and we determine the fixed points of $\hat{S}\left(S, q_{1}^{i}\right)=\sum_{i=1}^{n} s^{i}\left(S, q_{1}^{i}\right)$. We draw the reader's attention on the fact that we describe the second period sub-game equilibria focusing on level of inventories lower than the quantity $q^{m}$ a monopoly minimizing its cost of production would produce, where $q^{m}=(a-c) / 2$. Indeed, due to the presence of the interest rate $\rho$ and of $n-1$ competitors, no firm stores more than the quantity a monopoly producing in second period would sell, and all the sub-games that follow inventory choices larger than $q^{m}$ for all firms are trivially dominated. This restriction turns out to be useful when aggregating the individual best replies to derive the aggregate sales at the Nash equilibrium. To each fixed point of $\hat{S}\left(S, q_{1}^{i}\right)$ corresponds a unique n-tuple of equilibrium individual sales, and the conditions on inventories vectors leading to each of the equilibrium sales appear to be linked to conditions on the marginal revenues and on the marginal costs, as we now discuss.

Let us give an example of conditions on inventories leading to a particular Nash equilibrium in the sales sub-game. To be in a sub-game equilibrium in which firms $1,2, \ldots, k$ sell exactly their inventories, and firms $k+1, \ldots, n$ sell strictly more than 
their inventories, it suffices that given equilibrium sales of competitors,

(1) the marginal revenue of firm $k+1$ when selling $q_{1}^{k+1}$ is strictly higher than the marginal cost $c$ (so will it be for firms $k+2, \ldots, n$ who own inventories smaller than firm $k+1$ ),

(2) the marginal revenue of firm $k$ when selling $q_{1}^{k}$ is strictly lower than $c$ (so will it be for firms $1, \ldots, k-1$ who own inventories larger than firm $k$ ) and

(3) the marginal revenue of firm 1 when selling $q_{1}^{1}$ is strictly positive (and so will it be for firms $2, \ldots, k)$.

Indeed, when only firms $i=1, \ldots, k$ sell exactly their inventories, then in equilibrium firms $k+1, \ldots, n$ sell the same quantity higher than their inventories. Firms $k+1, \ldots, n$ are therefore confronted to the same equilibrium sales of competitors (equal to the sum of inventories of firms $1, \ldots, k$ plus $(n-1)$ times the quantity sold by any of the firms $k+1, \ldots, n)$. Consequently the marginal revenues of firms $k+1, \ldots, n$ are identical functions of individual sales $s$. Due to the fact that the marginal revenue is decreasing in individual sales $s$, then if firm $k+1$ (with the highest level of inventories $q_{1}^{k+1}$ in the group of firms $\{k+1, \ldots, n\}$ selling more than their inventories) faces a marginal revenue higher than the effective marginal cost at $s=q_{1}^{k+1}$, then the same is true for all the other firms of this group, explaining condition (1).

On the contrary, the marginal revenues of firms $1, \ldots, k$ (who sell exactly their inventories) evaluated at the equilibrium sales of their competitors jump upward the higher the level of firm inventories. Indeed firm $i$ is confronted to aggregate sales in equilibrium equal to the sum of inventories of firms $1, \ldots, i-1, i+1, \ldots k$, plus the identical sales of firms $k+1, \ldots, n$. This quantity is lower the higher the level of inventories of firm $i$, since the aggregate level of inventories of competitors selling these inventories is mechanically lower. The value of the marginal revenue at $s^{i}=q_{1}^{i}$ is however lower the higher the level of inventories of the firm we consider, by definition of the marginal revenue ${ }^{11}$, which explains why focusing on the comparison between the marginal revenue of firm $k$ when selling $q_{1}^{k}$ and the marginal cost $c$ is sufficient (condition (2)).

\footnotetext{
${ }^{11}$ One replaces $-q_{1}^{i}$ by $-2 q_{1}^{i}$ in each expression.
} 
Finally under assumption (A.1), and restricting our attention to $q_{1}^{1} \leq(a-c) / 2$, condition (3) is verified as long as we consider $k<n$. However when we characterize the sub-game equilibrium in which all firms are selling exactly their inventories, i.e. such that the marginal revenue of firm $n$ is lower than $c$ when it sells exactly $q_{1}^{n}$, then condition (3) needs to be verified. If (3) is verified, then the marginal revenues of firms $1, \ldots, n-1$ are also positive, leading to the sub-game equilibrium we are searching for.

The same analysis can be done to characterize all the sub-game equilibria, in particular those in which some firms are selling exactly their inventories and some others less than their inventories. To present all the sub-game equilibria, we introduce the following set of notations. The sequence of sets $\{B(\ell)\}_{\ell=0, \ldots, 2 n}$ characterizes for each $\ell$ the values of inventories leading to a sales sub-game in which some firms are selling more than, some firms are selling exactly, and some firms are selling less than their inventories. We define this sequence as follows:

- $B(0)=\left\{q_{1} \mid m^{1}\left(q_{1}^{1},(n-1)(a-c) /(n+1)\right) \geq c\right\}$ denotes the set of inventories such that all firms are selling in second period strictly more than their inventories,

- For $\ell \in\{1, \ldots, n-1\}, B(\ell)$ denotes the set of inventories such that firms $1, \ldots, \ell$ are selling exactly their inventories and firms $\ell+1, \ldots, n$ are selling strictly more. It is defined as

$$
\begin{aligned}
& B(\ell)=\left\{q_{1} \mid m^{\ell}\left(q_{1}^{\ell},(n-\ell)\left(a-c-\sum_{i=1}^{\ell} q_{1}^{i}\right) /(n-\ell+1)+\sum_{i=1}^{\ell-1} q_{1}^{i}\right)<c,\right. \\
& \left.m^{\ell+1}\left(q_{1}^{\ell+1},(n-\ell-1)\left(a-c-\sum_{i=1}^{\ell} q_{1}^{i}\right) /(n-\ell+1)+\sum_{i=1}^{\ell} q_{1}^{i}\right) \geq c\right\}
\end{aligned}
$$

- $B(n)=\left\{q_{1} \mid m^{n}\left(q_{1}^{n}, \sum_{i=1}^{n-1} q_{1}^{i}\right)<c, m^{1}\left(q_{1}^{1}, \sum_{i=2}^{n} q_{1}^{i}\right) \geq 0\right\}$ denotes the set of inventories such that all firms are selling exactly their inventories,

- For $\ell \in\{n+1, \ldots, 2 n-1\}, B(\ell)$ denotes the set of inventories such that firms $1, \ldots, \ell-n$ sell less than their inventories and firms $\ell-n+1, \ldots, n$ sell exactly their inventories. It is defined as

$$
\begin{aligned}
B(\ell)= & \left\{q_{1} \mid m^{\ell-n}\left(q_{1}^{\ell-n},(\ell-n-1)\left(a-\sum_{i=\ell-n+1}^{n} q_{1}^{i}\right) /(\ell-n+1)+\sum_{i=\ell-n+1}^{n} q_{1}^{i}\right)<0,\right. \\
& \left.m^{\ell-n+1}\left(q_{1}^{\ell-n+1},(\ell-n)\left(a-\sum_{i=\ell-n+1}^{n} q_{1}^{i}\right) /(\ell-n+1)+\sum_{i=\ell-n+2}^{n} q_{1}^{i}\right) \geq 0\right\}
\end{aligned}
$$


- Finally $B(2 n)=\left\{q_{1} \mid m^{n}\left(q_{1}^{n},(n-1) a /(n+1)\right)<0\right\}$ denotes the set of inventories such that all firms sell less than their inventories.

As its proof shows (available in appendix A.), this set of notations is sufficient to establish the following proposition.

Proposition 1 Under assumption (A.1) restricting the attention to inventories lower than the quantity a monopoly minimizing its costs would produce, $q_{1}^{1} \leq(a-c) / 2$, aggregate sales $S^{*}$ at the Nash equilibrium are given by:

1. if $q_{1} \in B(0)$, then all firms sell more than their inventories, and $S^{*}(0)=\frac{n(a-c)}{n+1}$,

2. if $q_{1} \in B(\ell)$ for $\ell \in\{1, \ldots, n-1\}$, then firms 1 to $\ell$ sell exactly their inventories and

$$
S^{*}(\ell)=\frac{(n-\ell)(a-c)+\sum_{i=1}^{\ell} q_{1}^{i}}{n-\ell+1}
$$

3. if $q_{1} \in B(n)$ then all firms sell exactly their inventories and $S^{*}(n)=\sum_{i=1}^{n} q_{1}^{i}$,

4. if $q_{1} \in B(\ell)$ for $\ell \in\{n+1, \ldots, 2 n-1\}$, then firms 1 to $\ell-n$ sell less than their inventories and

$$
S^{*}(\ell)=\frac{(\ell-n) a+\sum_{i=\ell-n+1}^{n} q_{1}^{i}}{\ell-n+1},
$$

5. if $q_{1} \in B(2 n)$ then all firms sell less than their inventories and $S^{*}(2 n)=\frac{n a}{n+1}$.

As for a given level of industry sales there is a unique corresponding level of individual sales, there is a unique vector of individual sales in equilibrium. Individual sales in equilibrium are given in the corollary at the end of the proof of proposition 1 in appendix A. We turn now to the analysis of the equilibrium of the game.

\section{Inventories in equilibrium and effect of entry}

To start with, we state what cannot be an equilibrium of our game, to determine on which of the regions $\{B(\ell)\}_{\ell=0, \ldots, 2 n}$ we can focus the search for a Nash equilibrium in pure strategies, if it exists. The lemma below shows first that firms cannot store in equilibrium more than what they sell in second period: as inventories are costly to produce, firms are strictly better off storing exactly what they sell. Second, the firm with the smallest level of inventories cannot store in equilibrium, as it is better off waiting for the second period to produce. 
Lemma 1 Any n-tuple of inventories $q_{1}=\left(q_{1}^{1}, \ldots, q_{1}^{n}\right)$ such that:

(i) some firms are selling strictly less than their inventories, i.e. $q_{1} \in B(\ell)$ for $\ell=$ $n+1, \ldots, 2 n$,

(ii) firms who are selling strictly more than their inventories are keeping strictly positive inventories,

cannot be an equilibrium.

Proof. Since inventories are costly to produce, if they were selling strictly less than their inventories, firms would be strictly better off reducing unilaterally their inventories. Situations in which some firms are selling less than their inventories cannot be an equilibrium: (i) holds for any value of $\rho$ non negative.

To prove (ii) let us examine the profit of firms selling strictly more than their inventories. Consider $q_{1} \in B(\ell)$ : the total profit of firms $i=\ell+1, \ldots, n$ is given by $\pi^{i}(\ell)=\left(a-S^{*}(\ell)\right) s^{i *}(\ell)-(1+\rho) c q_{1}^{i}-c\left(s^{i *}(\ell)-q_{1}^{i}\right)=\left(a-c-S^{*}(\ell)\right) s^{i *}(\ell)-\rho c q_{1}^{i}$. As $S^{*}(\ell)$ and $s^{i *}(\ell)$ are independent of $q_{1}^{i}$ for $i=\ell+1, \ldots, n$, this profit is strictly decreasing in $q_{1}^{i}$. Remark that from the expression of the bounds of the region in which firms 1 to $\ell$ are selling exactly their inventories, it is needed that $q_{1}^{\ell+1} \leq$ $\left(a-c-\sum_{i=1}^{\ell} q_{1}^{i}\right) /(n-\ell+1)$. This condition is trivially satisfied for $q_{1}^{\ell+1}=0$ and consequently the deviation to $q_{1}^{i}=0$ for $i=\ell+1, \ldots, n$ belongs to $B(\ell)$. (ii) holds: no firm selling more than its inventories can keep positive inventories in equilibrium. This property of the equilibrium holds also for firm $n$ when firms 1 to $n-1$ sell exactly their inventories: this firm is better off minimizing its cost by storing nothing.\|

This lemma insures that the search for an equilibrium can be restricted to n-tuple of inventories $\left(q_{1}^{1}, \ldots, q_{1}^{n}\right)$ such that in the sub-games that follow, a group of firms is selling exactly its inventories while rivals are selling strictly more. Moreover it also proves it is cost minimizing for firms selling strictly more than their inventories not to store at all. This applies in particular to firm $n$ when firms $1, \ldots, n-1$ store in first period. Consequently the search for an equilibrium can be restricted to n-tuple of inventories $\left(q_{1}^{1}, \ldots, q_{1}^{n}\right)$ belonging to region $B(\ell)$ for $\ell=1, \ldots, n-1$, with inventories $\left(q_{1}^{\ell+1}, \ldots, q_{1}^{n}\right)$ being equal to 0 . 
Theorem 1 below and its corollary characterize the equilibria of the game. For each number of firms competing over the two periods, and for every value of $(a, c, \rho)$ satisfying (A.1), these two results determine the n-tuple of inventories $\left(q_{1}^{1 *}, \ldots, q_{1}^{n *}\right)$ stored by firms at the Nash-perfect equilibrium of the game. Equilibria are such that a particular level of inventories are kept by $\ell$ firms, while $n-\ell$ are not storing, under certain conditions on $n$ and on the model parameters. The number of firms storing, $\ell$, and the individual inventories these firms keep, vary.

The proof of these two results is long and fastidious ${ }^{12}$. It consists in four steps. First (step 1) we determine the conditions under which the Cournot-Nash outcome is an equilibrium. Then (step 2) we determine the equilibrium values of inventories and the conditions on the model parameters such that $\ell$ firms store. More specifically to be a sub-game perfect equilibrium in which firms $1, \ldots, \ell$ store and firms $\ell+1, \ldots, n$ do not, a n-tuple of inventories $q_{1}^{*}(\ell) \equiv\left(q_{1}^{1 *}, \ldots, q_{1}^{i *}, \ldots, q_{1}^{\ell *}, 0, \ldots, 0\right)$ must be such that:

(a) There is no profitable individual deviation from $q_{1}^{*}(\ell)$ within $B(\ell): q_{1}^{*}(\ell)$ is more profitable than $\left(q_{1}^{1 *}, \ldots, q_{1}^{i-1 *}, q_{1}^{i}, q_{1}^{i+1 *}, \ldots, q_{1}^{\ell *}, 0, \ldots, 0\right)$ for any $q_{1}^{i}$ such that $q_{1} \in B(\ell)$ and for any firm $i=1, \ldots, \ell$.

(b) No firm storing finds profitable to reduce inventories down to 0: $q_{1}^{*}(\ell)$ is more profitable than $\left(q_{1}^{1 *}, \ldots, q_{1}^{i-1 *}, 0, q_{1}^{i+1 *}, \ldots, q_{1}^{\ell *}, 0, \ldots, 0\right)$ for any firm $i \in\{1, \ldots, \ell\}$. That is, firm $i$ must not find profitable to join the group of firms producing in second period only, to react optimally in period 2 to the inventories of the firms storing: (b) determines a condition under which at least $\ell$ firms store.

(c) No firm not storing in first period finds profitable to increase inventories to join the group of firms storing: $q_{1}^{*}(\ell)$ is more profitable than $\left(q_{1}^{1 *}, \ldots, q_{1}^{\ell *}, 0, \ldots, 0, q_{1}^{i}, 0, \ldots, 0\right)$ for any $q_{1}^{i}$ such that $q_{1} \in B(\ell+1)$ and for any firm $i \in\{\ell+1, \ldots, n\}$. That is (c) determines a condition under which at most $\ell$ firms store.

The conditions obtained in stages $(a),(b)$ and $(c)$ of step 2 are conditions on the cost of storage $\rho$, and the model parameters $a$ and $c$, that permits a given number of firms $\ell$ to store given the number of firms in competition $n$. More precisely an upper and a lower bound on the cost of storage ${ }^{13}$ are determined. These bounds

\footnotetext{
${ }^{12}$ See web appendix.

${ }^{13}$ More specifically the cost of storage relative to the difference between the demand intercept and the marginal cost of production, $\rho c /(a-c)$.
} 
are both functions of the number of firms $n$ in competition and the number of firms storing $\ell$. If the cost of storage exceeds the lower bound, then at most $\ell$ firms keep positive inventories, while if the cost of storage is lower than the upper bound then at least $\ell$ firms keep positive inventories. The level of inventories considered must also be an optimum for the firms that keep it, and for exactly $\ell$ firms to store these inventories, the cost of storage must be in between the two bounds. Then (step 3) proves that the conditions on model parameters determined in step 2 do not define an empty subset, and finally step 4 verifies that all possible values of the parameters have been covered, hence ensuring the existence of an equilibrium.

Theorem 1 The game possesses an equilibrium in pure strategies for any $n \geq 2$, and $a, c$, and $\rho$ satisfying (A.1).

Proof: See appendix B.\|

Corollary 1 It exists two sequences $\mathcal{U} \equiv\{U(n, \ell)\}_{\ell=1, \ldots, n-2}$ and $\mathcal{L} \equiv\{L(n, \ell)\}_{\ell=1, \ldots, n-2}$, with $U(n, \ell) \geq L(n, \ell)$ for any $\ell=1, \ldots, n-2$, given by

$$
L(n, \ell) \equiv \frac{(n-\ell+1-2 \sqrt{n-\ell})}{(n-\ell+1)(2 \ell \sqrt{n-\ell}+n-2 \ell)}
$$

and

$$
U(n, \ell) \equiv \frac{(n-\ell+2-2 \sqrt{n-\ell+1})}{(n-\ell+1)(\sqrt{n-\ell+1}(\ell-1)+n-\ell+2)},
$$

which are such that:

- for $\frac{\rho c}{a-c} \geq U(n, 1)$, the $n$-tuple $(0, \ldots, 0)$ is an equilibrium,

- for any $\ell=1, \ldots, n-2$ such that $L(n, \ell) \leq \frac{\rho c}{a-c} \leq U(n, \ell)$, the $n$-tuple $\left(q_{1}^{1 *}, \ldots, q_{1}^{n *}\right)$ where $q_{1}^{i *}=q_{1}^{*}(\ell)=\frac{a-c-(n-\ell+1) \rho c}{\ell+1}$ for $i=1, \ldots, \ell$ and $q_{1}^{i *}=0$ for $i=\ell+1, \ldots, n$ is an equilibrium,

- for $\frac{\rho c}{a-c} \leq L(n, n-2)$, the $n$-tuple $\left(q_{1}^{*}(n-1), \ldots, q_{1}^{*}(n-1), 0\right)$ is an equilibrium. 
Proof: See appendix B.\|

The number of firms storing at equilibrium results from the trade-off between a larger market share, obtained endogenously by an increase in inventories, and a smaller margin due to the increase in competition and in the cost of operations.

The first case presented in the corollary above corresponds to the static CournotNash equilibrium, that occurs if the cost of storage $\rho$ is large enough compared to the model parameters $a$ and $c$, given the intensity of competition. The second case corresponds to a situation where a subset of $\ell$ firms is storing, while $n-\ell$ are not. As we have shown in the proof of the theorem and its corollary, there may be multiple equilibria for the same values of the model parameters: indeed $U(n, \ell)$ determines an upper bound on $\rho$ below which at least $\ell$ firms are storing, while $L(n, \ell)$ determines a lower bound on $\rho$ above which at most $\ell$ firms store. Since $U(n, \ell+1) \geq L(n, \ell)$, several possibilities for the number of firms storing and for individual inventories at equilibrium may co-exist for a given value of the model parameters $(a, c, \rho)$. Moreover equilibria are asymmetric even if all firms are identical ex-ante: each firm that stores sells individually more than what a firm that does not store does. Finally the last case corresponds to the extreme situation of a very small cost of storage relative to the other model parameters and the intensity of competition, such that all firms store the product, apart one which prefers to wait the second period to produce and sell.

Let us characterize some properties of the equilibria compared to the static Cournot-Nash equilibrium. Since the market concentration is larger than what it would be if storage were not possible, for the same model parameters and the same number of firms in competition, the Herfindahl-Hirschman Index (HHI) of concentration should be larger when firms are able to store than when they are not, i.e. larger in our game than in static Cournot competition (everything else equal). Moreover competition should be fiercer. Indeed we have:

Proposition 2 When firms hold inventories, the price-cost margin is strictly lower, and the Herfindhal-Hirschman Index is striclty higher, than the value they obtain in static Cournot competition.

Proof: First let us prove formally that the market is more competitive than a static Cournot market. Using corollary 1 and proposition 1, the aggregate quantity sold 
when inventories are strictly positive is given by

$$
\begin{aligned}
S^{*} & =\frac{\ell(a-c-(n-\ell+1) \rho c)}{(\ell+1)(n-\ell+1)}+\frac{(n-\ell)(a-c)}{n-\ell+1} \\
& =\frac{\left(n(\ell+1)-\ell^{2}\right)(a-c)}{(\ell+1)(n-\ell+1)}-\frac{\ell \rho c}{\ell+1}
\end{aligned}
$$

that can be compared to $S^{C N}=\frac{n(a-c)}{n+1}$, the aggregate sales in static Cournot competition. It comes:

$$
\begin{aligned}
S^{*} \geq S^{C N} & \Leftrightarrow \frac{\left(n(\ell+1)-\ell^{2}\right)(a-c)}{(\ell+1)(n-\ell+1)}-\frac{n(a-c)}{n+1} \geq \frac{\ell \rho c}{\ell+1} \\
& \Leftrightarrow \frac{n(\ell+1)-\ell^{2}}{\ell(n-\ell+1)}-\frac{n(\ell+1)}{\ell(n+1)} \geq \frac{\rho c}{a-c} \\
& \Leftrightarrow \frac{(n+1)\left(n(\ell+1)-\ell^{2}\right)-(n-\ell+1) n(\ell+1)}{\ell(n-\ell+1)(n+1)} \geq \frac{\rho c}{a-c} \\
& \Leftrightarrow \frac{\ell(\ell+1) n-\ell^{2}(n+1)}{\ell(n-\ell+1)(n+1)} \geq \frac{\rho c}{a-c} \Leftrightarrow \frac{(\ell+1) n-\ell(n+1)}{(n-\ell+1)(n+1)} \geq \frac{\rho c}{a-c} \\
& \Leftrightarrow \frac{n-\ell}{(n-\ell+1)(n+1)} \geq \frac{\rho c}{a-c} .
\end{aligned}
$$

This condition is exactly the one that must be satisfied for $q_{1}^{*}(\ell)$ to belong to $B(\ell)$ obtained in (38); consequently the aggregate sales of the industry when firms hold inventories are strictly larger than the aggregate sales obtained in static Cournot competition. Hence the market price and the price-cost margin are smaller than in static Cournot competition ${ }^{14}$.

Second, the HHI is larger when firms hold inventories than in static Cournot competition. Indeed due to the fact that firms are identical, the equilibrium would be symmetric in static Cournot competition, and market shares identical (equal to $1 / n)$. As we proved in corollary 1 , market shares differ across group of firms when the output is stored, and the equilibrium is asymmetric. As the HHI is the sum of squared market shares, this grants immediately that the HHI is larger when firms hold inventories than in static Cournot competition, where it reaches its minimum.\|.

As we already explained, increasing inventories to credibly commit to gain market shares has a larger profitability when a few firms compete on the market, while an increase in the number of competitors reduces the margin and hence reduces the

\footnotetext{
${ }^{14}$ See Boyer and Moreaux ([1985], [1986]) for a similar result in the context of a Stackelberg oligopoly.
} 
incentive to acquire market shares by investing in inventories. This suggests a non monotonicity of the equilibrium in the number of competitors. We now confirm this intuition:

Proposition 3 The number of firms storing $\ell$ and the industry inventories $\ell q_{1}^{*}(\ell)$ may be non-monotonic in the number of firms in competition $n$.

Proof. Let us examine the values of the bounds on $\rho c /(a-c)$ established for the extreme cases, $\ell=1$ and $\ell=n-2$. We have:

$$
\begin{aligned}
& U(n, 1)=\frac{n+1-2 \sqrt{n}}{n(n+1)} \\
& L(n, 1)=\frac{n-2 \sqrt{n-1}}{n(2 \sqrt{n-1}+n-2)} \\
& U(n, n-2)=\frac{4-2 \sqrt{3}}{3((n-3) \sqrt{3}+4)} \\
& L(n, n-2)=\frac{3-2 \sqrt{2}}{3(2(n-2) \sqrt{2}-n+4)} .
\end{aligned}
$$

It is immediate to check that the lower bounds given by (12) and (13) are strictly decreasing with respect to $n$. Moreover it is possible to plot as a function of $n$ the upper bounds given by (10) and (11) with a mathematical software (e.g. Maxima or Mathematica), for values $n \geq 2$, to prove that it is non monotonic (concave) in $n$. By continuity of $U(n, \ell)$ and $L(n, \ell)$ with respect to $\ell$, there is a subset of values for $\ell$ in $1, \ldots, n-2$ for which $U(n, \ell)$ and $L(n, \ell)$ are non monotonic in $n$. Therefore, for a given value of $\rho c /(a-c)$, the number of firms storing at equilibrium, that results from the comparison of the sequences $\mathcal{U}$ and $\mathcal{L}$ with $\rho c /(a-c)$, may be nonmonotonic in the number of firms in competition, $n$. It follows that the aggregate inventories in equilibrium $\ell q_{1}^{*}(\ell)$ can also be non-monotonic in $n$.\|

It is possible to study the effect of an entry of competitors on the market by using the corollary of Theorem 1 to compute in an example the number of firms storing at equilibrium, the aggregate inventories kept by the industry, the aggregate sales of firms that do not store, the aggregate industry sales, and compare these values to the outcome of static Cournot competition when the number of competitors changes. The table below produces an example for $a=2.5, c=1$, and $\rho=0.03$, generated with a spreadsheet software. 


\section{[INSERT TABLE 1 HERE]}

Let us briefly describe the content of the table. Column (1) indicates the number of firms competing on the market, column (2) (respectively (3)) the minimal (resp. maximal) number of firms storing at equilibrium. These numbers are directly coming from the conditions on $\rho$ expressed in the corollary, and whenever they are identical we suppressed the one in column $(2)^{15}$. Then column (4) (resp. (5)) presents the aggregate level of inventories stored by the minimal (resp. maximal) number of firms storing, while column (6) (resp. (7)) shows the aggregate sales of the firms that are not storing when the number of firms storing is minimum (resp. maximum). Column (8) (resp. (9)) presents the total sales of the industry when the number of firms storing is minimal (resp. maximal), that can be compared to the aggregate sales a static Cournot oligopoly would realize, computed in column (10) and equal to $n(a-c) /(n+1)$.

As the table presents in columns (2) and (3), there may be multiple equilibria. Moreover as column (4) and (5) show, the minimal and the maximal number of firms storing at equilibrium, as well as the corresponding aggregate inventories of the industry, are non monotonic in the number of firms in competition $n$. That is when the number of firms in competition increases, the number of firms storing and the level of aggregate inventories both increase, to then decrease when the number of firms in competition is large enough. This illustrates the trade-off we described: the profitability of exerting some leadership by storing decreases the larger the number of firms in competition for a given cost of storage. When the number of firms is large enough, all the gains in volume obtained through leadership are offset by losses in margin over a marginal cost that includes the cost of storage. In that case the unique equilibrium is the Cournot-Nash one. This feature implies that the aggregate level of inventories is non-monotonic in the number of competitors on the market. For example when $n=6$, there can be up to 3 firms storing, and industry sales equal to 1.384 (in column (9)) are $7 \%$ higher than 1.286 , the value they reach in static Cournot competition (column (10)). For $n=13$, up to 5 firms store and sales equal to 1.447 are $3.8 \%$ higher than in static Cournot competition. For $n=19$, up to 3 firms are storing and sales equal to 1.455 are $2 \%$ higher than in static Cournot competition.

\footnotetext{
${ }^{15}$ It is replaced with a - ; the same convention applies to the rest of the table.
} 
We also compute the Herfindahl-Hirschman Index (HHI) when the number of firms storing is minimal (resp. maximal), presented in column (11) (resp. (12)), that can be compared to the HHI at the Cournot-Nash equilibrium (column (13)). Competition and concentration may therefore simultaneously increase when the number of firms decreases from perfect competition to oligopolistic market structure, and are clearly larger than their values in Cournot competition. In the example for $n=6$, the HHI is $25 \%$ higher in our model than in Cournot ( $H H I=2076$ instead of 1666), and twice higher for $n=19$ (HHI= 1155 instead of 526)! Comparing our results to the HHI thresholds from the U.S. Horizontal Mergers Guidelines ${ }^{16}$, the static Cournot market with $n=10$ firms would be unconcentrated $(H H I=1000)$, while if 3 firms store as it is potentially the case at equilibrium, the $H H I=1619$ would make it fall into the category of moderately concentrated markets. Competition is nonetheless fiercer.

We conclude this section by addressing two comments, first on the role of assumption (A.1), second on the role of a small interest rate. If we relax (A.1) to consider any type of market demand, we would still get asymmetric equilibria. However there may be an additional upper limit on individual inventories to consider, in addition to the conditions in the sets $B(\ell)$ we presented ${ }^{17}$.

By introducing the interest rate, we made first period production slightly more costly than second period production. This allows us to rule out of the set of equilibria the weakly dominated ones. If there were no interest rate, the ultimate follower would be indifferent between producing in period 1 and producing in period 2 , and if this firm produces the $n$-firms Cournot production in period 1 , then all the other firms are also better off producing a Cournot outcome in period 1. The result we would obtain in our game would be a generalization to the case of an oligopoly

\footnotetext{
${ }^{16}$ of August 19, 2010

${ }^{17}$ In the duopoly game with $\rho$ close to 0 , this level is equal to the intersection between the curves $q_{1}^{1}=\frac{a}{2}-\frac{1}{2} q_{1}^{2}$ and $q_{1}^{2}=\frac{a-c}{2}-\frac{1}{2} q_{1}^{1}$ that is $\left(q_{1}^{1}, q_{1}^{2}\right)=\left(\frac{a+c}{3}, \frac{a-2 c}{3}\right)$. The role of assumption (A.1) is to rule out credible threats of putting leaders with redundant inventories, by forcing prices to be lower than the marginal cost when a follower increases its inventories to force the leader to sell less than its inventories. Although Arvan [1985] identifies the possibility for a leader to be put with redundant inventories in his duopoly setting, he does not show that for this threat to modify the behaviour of the leader, it must be the case that the market price stays at a level such that the follower realizes a non-negative profit. The fact that redundant production may matter has not been analyzed by Saloner [1987], Pal ([1991], [1996]).
} 
of the result obtained for a duopoly by Saloner [1987], who shows that any pair of inventories on the outer envelope of the Cournot reaction functions between the two Stackelberg outcomes ${ }^{18}$ is an equilibrium in pure strategies. The interest rate (or a cost of storage) is a selection device that forces the ultimate follower to wait until period 2 to produce: this can be seen as a special case of the selection criterium presented in the duopoly game by Pal ([1991], [1996] $)^{19}$, who consider more generally cost differentials between periods. In an oligopoly the cost of storage is the economic force that determines how many firms store and exert some leadership amongst all the market competitors.

\section{Conclusion}

Without any other incentive than strategic to store, and in a perfectly symmetric setting, this paper shows that it is possible to observe positive level of inventories and asymmetric market shares at the equilibrium of a two-period Cournot oligopolistic market, no matter the finite number of firms in competition. The rationale for storing lies in the fact that inventories may be used to exert some market share leadership, but at the cost of reduced margins. The main intuition is that by dumping the cost of production of inventories in first period, firms obtain an endogenous but fixed capacity from which they can sell at zero marginal cost in second period. This marginal cost advantage translates into the search for leadership in our setting of oligopolistic quantity competition with perfect substitutes: firms choose a level of inventories such that their market share is increased compared to the static situation, competition is fiercer than when storage is impossible or the interaction is static, and concentration is also larger. The number of firms that store at equilibrium varies with the number of firms in competition, but as some firms store before selling while the others produce and sell simultaneously, our paper suggests that "make-to-stock" and "make-to-order" organizations of the operations may co-exist at the Nash equilibrium of an oligopolistic interaction on the final market. Finally an example illustrates the fact that observing larger industry inventories and a larger

\footnotetext{
${ }^{18}$ Under some extra assumptions on demand and cost missing in Saloner's work relating to assumption (A.1) and to the fact that leaders may be put with redundant production, as we argued before.

${ }^{19}$ See also Robson [1990].
} 
number of firms storing is more likely under an oligopolistic market structure than under market structures closer to the duopoly or to perfect competition: the number of firms storing and the aggregate inventories are non monotonic in the number of market participants.

\section{Appendix}

\section{A. Proof of proposition 1}

The proof is done in 3 steps. (Step 1) shows how to simplify each firm second period problem to enlighten the role of period 1 production (i.e. inventories) and derives the individual sales each firm chooses as a best reply to the aggregate sales of its competitors, $\widehat{s}^{i}\left(S^{-i}, q_{1}^{i}\right)$, which depends on inventories. This step has already been completed at the beginning of section 3. It establishes that the best response of any firm $i, \widehat{s}^{i}\left(S^{-i}, q_{1}^{i}\right)$, is given by equation (7). Bear in mind that as we study an oligopolistic competition, it is possible to restrict one's attention to period 1 inventories lower or equal to the individual production of a monopoly minimizing its cost of production, denoted $q^{m}$. Given the opportunity cost of producing in period 1 instead of period 2, and given the demand and costs parameters assumed before, this quantity is equal to $q^{m}=(a-c) / 2$. Consequently, the vector of period 1 production is such that $q_{1} \in[0,(a-c) / 2]^{n}$.

In Step 2, we aggregate all the best replies to find the equilibrium aggregate sales of the industry. To do so, we construct the best reply of each firm to the aggregate quantity sold by the industry, $\widehat{s}^{i}\left(S, q_{1}^{i}\right)$, and we sum these functions over all firms to obtain the industry best reply to an aggregate sales level, $\sum_{i \in I} \widehat{s}^{i}\left(S, q_{1}^{i}\right)=\widehat{S}\left(S, q_{1}\right)$. To construct the best reply $\widehat{s}^{i}\left(S, q_{1}^{i}\right)$, also known as the backward reaction mapping (from Novshek 1984 terminology) we first determine the cumulative reaction to $S^{-i}$ for firm $i, S_{i}^{\bullet}\left(S^{-i}, q_{1}^{i}\right)=\left\{s^{i}+S^{-i} / s^{i}=\widehat{s}^{i}\left(S^{-i}, q_{1}^{i}\right)\right\}$.

$$
S_{i}^{\bullet}\left(S^{-i}, q_{1}^{i}\right)= \begin{cases}\frac{1}{2}\left(a-c+S^{-i}\right) & \text { if } S^{-i} \leq a-c-2 q_{1}^{i} \\ q_{1}^{i}+S^{-i} & \text { if } S^{-i} \in\left[a-c-2 q_{1}^{i}, a-2 q_{1}^{i}\right] \\ \frac{1}{2}\left(a+S^{-i}\right) & \text { if } S^{-i} \geq a-2 q_{1}^{i}\end{cases}
$$

Then we invert it to obtain the inverse cumulative best response function $S_{i}^{-i}\left(S, q_{1}^{i}\right)$ 
for firm $i$. As $S_{i}^{\bullet}\left(S^{-i}, q_{1}^{i}\right)$ is strictly increasing it has a unique inverse,

$$
S^{-i}\left(S, q_{1}^{i}\right)= \begin{cases}2 S-(a-c) & \text { if } S \in\left[\frac{a-c}{2}, a-c-q_{1}^{i}\right] \\ S-q_{1}^{i} & \text { if } S \in\left[a-c-q_{1}^{i}, a-q_{1}^{i}\right] \\ 2 S-a & \text { if } S \geq a-q_{1}^{i}\end{cases}
$$

Finally we solve for the individual sale $s^{i}$ in $\left\{s^{i} / S-s^{i}=S^{-i}\left(S, q_{1}^{i}\right)\right\}$, to obtain the backward reaction mapping,

$$
\widehat{s}^{i}\left(S, q_{1}^{i}\right)= \begin{cases}(a-c)-S & \text { if } S \in\left[(a-c) / 2, a-c-q_{1}^{i}\right] \\ q_{1}^{i} & \text { if } S \in\left[a-c-q_{1}^{i}, a-q_{1}^{i}\right] \\ a-S & \text { if } S \geq a-q_{1}^{i}\end{cases}
$$

where as in (7), given some industry sales $S$, firm $i$ can either sell more than (first line of (16)), or sell exactly (second line of (16)), or sell less than (third line of (16)) its inventories $q_{1}^{i}$. Under assumptions (A.1) and under the convention that firm $i$ owns larger inventories than firm $i+1$, summing all the backward reaction mappings to obtain $\widehat{S}\left(S, q_{1}\right)$ can be done easily. Indeed for any level of industry sales $S$, all firms are either selling at least their inventories or selling at most their inventories, but it is not possible that some of them sell strictly more than their inventories, while some others are selling strictly less. To put it differently the cut-off values for $S$ determining the reaction of an individual firm in (16) are "nicely" ranked across firms. To see this, first remark that

$$
(a-c) / 2 \leq a-c-q_{1}^{1} \leq \ldots \leq a-c-q_{1}^{n} \leq a-c
$$

and

$$
a-q_{1}^{1} \leq \ldots \leq a-q_{1}^{n}
$$

As justified in step 1, there is no loss of generality to restrict our attention to period 1 productions lower than $q^{m}=(a-c) / 2$. Consequently the lower bound in the sequence of inequalities (18) can be minored, $a-q_{1}^{1} \geq(a+c) / 2$. It suffices to remark that as a consequence of (A.1), the upper bound of the sequence of inequalities (17) is lower than the lower bound of the sequence (18), i.e. $a-c \leq(a+c) / 2$, to be able to rank across all firms the cut-off values at which there are kinks in the backward reaction mappings $(16)$

$$
a-c-q_{1}^{1} \leq \ldots \leq a-c-q_{1}^{n} \leq a-q_{1}^{1} \leq \ldots \leq a-q_{1}^{n}
$$


For a level of industry sales $S$ lower than $a-c-q_{1}^{1}$, all firms are selling more than their period 1 production and consequently the sum of all the backward reaction mappings is simply equal to $n$ times the expression in the first line of $(16),(a-c)-S$. For $S$ higher than $a-c-q_{1}^{1}$ and lower than $a-c-q_{1}^{2}$, firm 1 is selling exactly its inventories and firms 2 to $n$ are selling strictly more: the sum of the backward reaction mappings is equal to $q_{1}^{1}$ plus $n-1$ times $(a-c)-S, \ldots$ and so on. For industry sales higher than $a-c-q_{1}^{n}$ and lower than $a-q_{1}^{1}$, all firms are selling exactly their inventories, and $\sum_{i \in I} \widehat{s}^{i}\left(S, q_{1}^{i}\right)=\sum_{i \in I} q_{1}^{i}=Q_{1}$. For $S$ higher than $a-q_{1}^{1}$ and lower than $a-q_{1}^{2}$ firm 1 sells less than its inventories and firms 2 to $n$ sell exactly their inventories: $\sum_{i \in I} \widehat{s}^{i}\left(S, q_{1}^{i}\right)$ is equal to $a-S+\sum_{i \geq 2} q_{1}^{i}, \ldots$ and so on to complete the summation. To summarize, $\widehat{S}\left(S, q_{1}\right)$ is given by

$$
\widehat{S}\left(S, q_{1}\right)= \begin{cases}n(a-c-S) & \text { if } S \in\left[(a-c) / 2, a-c-q_{1}^{1}\right] \\ (n-k)(a-c-S)+\sum_{i=1}^{k} q_{1}^{i} & \text { if } S \in\left[a-c-q_{1}^{k}, a-c-q_{1}^{k+1}\right] \\ & \text { for } k=1, \ldots, n-1 \\ \cdots & \ldots \\ \sum_{i=1}^{n} q_{1}^{i} & \text { if } S \in\left[a-c-q_{1}^{n}, a-q_{1}^{1}\right] \\ k(a-S)+\sum_{i=k+1}^{n} q_{1}^{i} & \text { if } S \in\left[a-q_{1}^{k}, a-q_{1}^{k+1}\right] \\ & \text { for } k=1, \ldots, n-1 \\ \cdots & \ldots \\ n(a-S) & \text { if } S \geq a-q_{1}^{n}\end{cases}
$$

Step 3 determines the fixed points of $\widehat{S}\left(S, q_{1}\right)$. As there are $2 n$ cut-off values determining the different expressions of $\widehat{S}$, there are $2 n+1$ different expressions and potentially $2 n+1$ different sub-game equilibria to find. We index the consecutive lines from $(20)$ by $\ell=0, \ldots, 2 n$ : at line 0 all firms sell more than their inventories, at line 1 firm 1 sells exactly its inventories and the others more,... and so on. At line $n$ all firms sell exactly their inventories and at line $n+1$ firm 1 sells less than its inventories while the others sell exactly their inventories, until line $2 n$. We derive the fixed points of $\widehat{S}\left(S, q_{1}\right)$ line by line: for every line $\ell=0, \ldots, 2 n$, there is a unique equilibrium aggregate sales level $S^{*}(\ell)$. To this equilibrium industry sales $S^{*}(\ell)$ corresponds a unique set of period 1 inventories $B(\ell)$ such that if the vector of firms inventories $q_{1}$ belongs to $B(\ell)$, then the equilibrium is $S^{*}(\ell)$. Let us describe these fixed points and the sets that are associated to them. The equilibrium 
in which all firms sell more than their inventories (line $\ell=0$ ) is characterized by $S^{*}(0)=n(a-c) /(n+1)$. Inventories must be such that $S^{*}(0) \leq a-c-q_{1}^{1}$ that is must belong to $B(0)$ given by

$$
B(0)=\left\{q_{1} \mid q_{1}^{1} \leq(a-c) /(n+1)\right\}
$$

For $\ell=1, \ldots, n-1$, equilibrium aggregate sales are $S^{*}(\ell)=((n-\ell)(a-c)+$ $\left.\sum_{i=1}^{\ell} q_{1}^{i}\right) /(n-\ell+1)$, and $q_{1}$ must belong to $B(\ell)$ given by

$$
B(\ell)=\left\{q_{1} \mid q_{1}^{\ell} \geq\left(a-c-\sum_{i=1}^{\ell-1} q_{1}^{i}\right) /(n-\ell+2), q_{1}^{\ell+1} \leq\left(a-c-\sum_{i=1}^{\ell} q_{1}^{i}\right) /(n-\ell+1)\right\} .
$$

For $\ell=n, S^{*}(n)=\sum_{i=1}^{n} q_{1}^{i}$ and $q_{1}$ must belong to $B(n)$

$$
B(n)=\left\{q_{1} \mid q_{1}^{n} \geq\left(a-c-\sum_{i=1}^{n-1} q_{1}^{i}\right) / 2, q_{1}^{1} \leq\left(a-\sum_{i=2}^{n} q_{1}^{i}\right) / 2\right\} .
$$

For $\ell=n+1, \ldots, 2 n-1, S^{*}(\ell)=\left((\ell-n) a+\sum_{i=\ell-n+1}^{n} q_{1}^{i}\right) /(\ell-n+1)$ and $q_{1} \in B(\ell)$ such that

$$
B(\ell)=\left\{q_{1} \mid q_{1}^{\ell-n} \geq\left(a-\sum_{i=\ell-n+1}^{n} q_{1}^{i}\right) /(\ell-n+1), q_{1}^{\ell-n+1} \leq\left(a-\sum_{i=\ell-n+2}^{n} q_{1}^{i}\right) /(\ell-n+2)\right\} .
$$

Finally for $\ell=2 n, S^{*}(2 n)=n a /(n+1)$ and $q_{1} \in B(2 n)$ such that

$$
B(2 n)=\left\{q_{1} / q_{1}^{n} \geq a /(n+1)\right\}
$$

The intersection between (the interior of) two sets is empty, $B(\ell) \bigcap B\left(\ell^{\prime}\right)=\emptyset$ for $\ell \neq \ell^{\prime}$, and the reunion of all sets $\bigcup_{\ell=0, \ldots, 2 n} B(\ell)$ encompasses exactly all the cases for $q_{1}$ we are interested in. We complete this proof by expressing the conditions on inventories in terms of conditions on the marginal revenues to obtain our result. Obviously,

$$
q_{1}^{1} \leq \frac{a-c}{n+1} \Leftrightarrow m^{1}\left(q_{1}^{1}, \frac{n-1}{n+1}(a-c)\right) \geq c
$$

and so on... it remains to use the backward reaction mapping of each firm to obtain individual sales:

Corollary 2 (to Proposition 1) For each equilibrium level of aggregate sales $S^{*}(\ell)$, $\ell=0, \ldots, 2 n$, there is a unique equilibrium vector of individual sales $s^{*}(\ell)$ given by:

1. if $q_{1} \in B(0), s^{i *}(0)=\frac{a-c}{n+1}$ for any $i \in I$, 
2. if $q_{1} \in B(\ell)$ for $\ell \in\{1, \ldots, n-1\}$, then $s^{i *}(\ell)=q_{1}^{i}$ for $i=1, \ldots, \ell$ and

$$
s^{i *}(\ell)=\frac{a-c}{n-\ell+1}-\frac{\sum_{i=1}^{\ell} q_{1}^{i}}{n-\ell+1} \text { for } i=\ell+1, \ldots, n,
$$

3. if $q_{1} \in B(n)$ then $s^{i *}(n)=q_{1}^{i}$ for all $i \in I$,

4. if $q_{1} \in B(\ell)$ for $\ell \in\{n+1, \ldots, 2 n-1\}$, then $s^{i *}(\ell)=q_{1}^{i}$ for $i=\ell-n+1, \ldots, n$ and

$$
s^{i *}(\ell)=\frac{a}{\ell-n+1}-\frac{\sum_{i=\ell-n+1}^{n} q_{1}^{i}}{\ell-n+1} \text { for } i=1, \ldots, \ell-n,
$$

5. if $q_{1} \in B(2 n)$ then $s^{i *}(2 n)=\frac{a}{n+1}$ for any $i \in I$. 


\section{References}

[1] Allaz, B., and Vila, J.-L. [1993]. Cournot competition, forward markets and efficiency. Journal of Economic Theory, vol. 59, no 1 : 1-16.

[2] Amihud, Y., and Mendelson, H. [1989]. Inventory behaviour and market power. International Journal of Industrial Organisation vol.7 : 269-280.

[3] Anderson, R. [1984]. The industrial organization of futures markets. LexingtonBooks, DC Heath, Lexington (Mass.).

[4] Arrow, K.J., T. Harris and J. Marschak. (1951). Optimal Inventory Policy. Econometrica, vol. 19, no. 3, p. 250-272.

[5] Arvan, L. [1985]. Some examples of dynamic Cournot duopoly with inventory. Rand Journal of Economics, vol.16, N4: 569-578.

[6] Arvan, L., and L. Moses. (1982). Inventory Investment and the Theory of the Firm. The American Economic Review, vol. 72, no 1, p. 186-193.

[7] Bagwell, K. [1991]. Commitment and observability in games. Games and Economic Behaviour, vol.8 : 271-280.

[8] Bamon, R., and Fraysse, J. [1983]. Existence of Cournot equilibrium in large markets. Econometrica, vol. 53, no 3 : 587-597.

[9] Blanchard, O.J. (1983). The Production and Inventory Behaviour of the American Automobile Industry. Journal of Political Economy, vol. 91, no. 3, p. 365400.

[10] Boyer, M., and Moreaux, M. [1985]. L'équilibre concurrentiel comme limite de suites d'équilibres stratégiques de Stackelberg, L'Actualité Economique, Revue d'Analyse Economique, vol. 61, No 3: 299-315.

[11] Boyer, M., and Moreaux, M. [1986]. Perfect competition as the limit of a hierarchical market game, Economic Letters, vol. 22, No 2-3: 115-118.

[12] Dixit, A. [1980]. The role of investment in entry-deterrence, The Economic Journal 90 (March) : 95-106. 
[13] Kirman, A., and Sobel, J. [1974]. Dynamic oligopoly with inventories. Econometrica, vol. 42, no 2 : 201-204.

[14] Liski, M., and Montero, J.-P. [2006]. Forward trading and collusion in oligopoly. Journal of Economic Theory, vol.131, no 1 : 212-230.

[15] Maggi, G. [1998]. The value of commitment with imperfect observability and private information. Rand Journal of Economics, vol.30(4) : 555-574.

[16] Mahenc, P., and Salanié, F. [2004]. Softening competition through forward trading. Journal of Economic Theory, vol.116, no 2: 282-293.

[17] Novshek, W. [1984]. Finding all n-firms Cournot Equilibria. International Economic Review, vol. 25, no 1: 61-70.

[18] Pal, D. [1991]. Cournot Duopoly with Two Production Periods and Cost Differentials. Journal of Economic Theory, vol.55 : 441-448.

[19] Pal, D. [1996]. Endogenous Stackelberg Equilibria with Identical Firms. Games and Economic Behavior, vol. 12 : 81-94.

[20] Robson, A. [1990]. Stackelberg and Marshall. The American Economic Review, vol. $80: 69-82$.

[21] Romano, R., and Yildirim, H. [2005]. On the endogeneity of Cournot-Nash and Stackelberg equilibria: games of accumulation. Journal of Economic Theory, vol. $120: 73-107$.

[22] Saloner, G. [1987]. Cournot Duopoly with Two Production Periods. Journal of Economic Theory, vol. 42 : 183-187.

[23] Schelling, T. [1960]. The Strategy of Conflict. Harvard University Press.

[24] Thille, H., and Slade, M. [2000]. Forward trading and adjustement costs in Cournot markets. Cahier d'Economie Politique, no 37 : 177-195.

[25] Thille, H. [2003]. Forward trading and storage in a Cournot duopoly. Journal of Economic Dynamics and Control, vol. 27, no 4 : 651-666. 
[26] Thille, H. [2006]. Inventories, Market Structure and Price Volatility. Journal of Economic Dynamics and Control, 30, 1081-1104.

[27] Van Damme, E., and Hurkens, S. [1997]. Games with imperfectly observable commitment. Games and Economic Behaviour, vol.21 : 282-308.

[28] Ware, R. [1984]. Sunk Costs and Strategic Commitment: a Proposed Threestage Equilibrium, The Economic Journal, vol. 94, No 374: 370-378.

[29] Ware, R. [1985]. Inventory Holding as a Strategic Weapon to Deter Entry, Economica, vol. 52: 93-101.

[30] Zipkin, P.H. (2000). Foundations of Inventory Management. McGraw-Hill. 


\section{Web appendix}

\section{B. Proof of theorem 1 and its corollary}

Lemma 1 insures that the search for an equilibrium can be restricted to n-tuple of inventories $\left(q_{1}^{1}, \ldots, q_{1}^{n}\right)$ such that in the sub-games that follow, a group of firms is selling exactly its inventories while the rest is selling strictly more. Moreover it is cost minimizing for firms selling strictly more than their inventories not to store at all. Finally it is also cost minimizing for firm $n$ not to store when all others keep inventories in first period. Let us recall the result obtained in proposition 1 and corollary 2: when $\ell$ firms store in first period, individual sales $s^{i *}(\ell)$ and aggregate sales $S^{*}(\ell)$ are respectively given by:

$$
\begin{aligned}
& s^{i *}(\ell)=q_{1}^{i} \text { for } i=1, \ldots, \ell \\
& s^{i *}(\ell)=\frac{a-c-\sum_{i=1}^{\ell} q_{1}^{i}}{n-\ell+1} \text { for } i=\ell+1, \ldots, n \\
& S^{*}(\ell)=\frac{(n-\ell)(a-c)+\sum_{i=1}^{\ell} q_{1}^{i}}{n-\ell+1} .
\end{aligned}
$$

For this sub-game equilibrium to occur, inventories must be such that $q_{1} \in B(\ell)$. Using the expression of the marginal revenues $m^{\ell}(.,$.$) and m^{\ell+1}(.,$.$) rewritten as in$ (22), this condition writes:

$$
\begin{aligned}
& q_{1}^{\ell} \geq \frac{a-c-\sum_{i=1}^{\ell-1} q_{1}^{i}}{n-\ell+2} \\
& q_{1}^{\ell+1} \leq \frac{a-c-\sum_{i=1}^{\ell} q_{1}^{i}}{n-\ell+1} .
\end{aligned}
$$

The proof consists of 4 steps. First (step 1) we determine the conditions under which the Cournot-Nash outcome is an equilibrium. Then (step 2) we determine equilibrium values of inventories and the conditions on the model parameters such that $\ell$ firms store. Then (step 3) proves that the conditions on model parameters determined in step 2 do not define an empty subset, and finally step 4 verifies that all possible values of the parameters have been covered, hence ensuring the existence of an equilibrium.

Step 1. Cournot-Nash equilibrium. 
Start with the case where no firm finds profitable to store. The profit oligopolists obtain when not storing is equal to

$$
\pi_{*}^{0}=\left(\frac{a-c}{n+1}\right)^{2}
$$

that is the Cournot-Nash profit. For this outcome to be an equilibrium, we need to check that parameters are such that no firm (say firm 1) finds profitable to increase inventories. When deviating, firm 1 has to select in $B(1)$ its preferred quantity. Using the expression from proposition 1, the deviation chosen by firm 1 must be such that $q_{1}^{1} \geq(a-c) /(n+1)$ and $q_{1}^{1} \leq a-c$. The profit when deviating in $B(1)$ to be maximized in $q_{1}^{1}$ is

$$
\pi^{1}=\left(a-\frac{(n-1)(a-c)+q^{1}}{n}-(1+\rho) c\right) q^{1}
$$

which has a maximum given by $q_{d e v 0}^{1}=(a-c-n \rho c) / 2$. The profit obtained when deviating is maximal at this quantity if $(a-c-n \rho c) / 2 \geq(a-c) /(n+1)$, which is equivalent to

$$
\rho c \leq \frac{(n-1)(a-c)}{n(n+1)},
$$

else the solution is to choose the deviation to the lower bound of the region $q_{1}^{1}=$ $(a-c) /(n+1)$, which is strictly dominated by waiting for the second period to produce and sell the (same) Cournot-Nash quantity. The profit obtained at the deviation $q_{d e v 0}^{1}$ is equal to:

$$
\pi_{d e v 0}^{1}=\left(\frac{a-c-n \rho c}{2 \sqrt{n}}\right)^{2}
$$

and the comparison with $\pi_{*}^{0}$ gives immediately that $\pi_{\text {dev } 0}^{1} \geq \pi_{*}^{0}$ if $\rho c \leq \frac{(n+1-2 \sqrt{n})(a-c)}{n(n+1)}$. Remark that this condition defines a bound on $\rho c$ that satisfies (33) above, as $n+$ $1-2 \sqrt{n} \leq n-1$ for $n \geq 2$. Therefore Cournot-Nash is an equilibrium of the game if

$$
\rho c \geq \frac{(n+1-2 \sqrt{n})(a-c)}{n(n+1)} .
$$

This condition ensures that no firm finds profitable to deviate from the n-tuple $(0, \ldots, 0)$, i.e. that at most 0 firms store.

Step 2. Equilibrium with $\ell$ firms storing. 
Let $\ell$ denote the identity of the last firm storing in $1, \ldots, n-1$. To be a sub-game perfect equilibrium in which firms $1, \ldots, \ell$ store and firms $\ell+1, \ldots, n$ do not, a n-tuple of inventories $\left(q_{1}^{1 *}, \ldots, q_{1}^{n *}\right)$ must be such that:

(a) There is no profitable individual deviation from $q_{1}^{*}$ within $B(\ell)$ :

$\left(q_{1}^{1 *}, \ldots, q_{1}^{i-1 *}, q_{1}^{i *}, q_{1}^{i+1 *}, \ldots, q_{1}^{\ell *}, 0, \ldots, 0\right)$ is more profitable than

$\left(q_{1}^{1 *}, \ldots, q_{1}^{i-1 *}, q_{1}^{i}, q_{1}^{i+1 *}, \ldots, q_{1}^{\ell *}, 0, \ldots, 0\right)$ for any $q_{1}^{i}$ such that $q_{1} \in B(\ell)$ and for any firm $i=1, \ldots, \ell$.

(b) No firm storing finds profitable to reduce inventories down to 0 (in that deviation this firm joins the group of firms producing in second period only and reacts optimally to the inventories of the firms storing):

$\left(q_{1}^{1 *}, \ldots, q_{1}^{i-1 *}, q_{1}^{i *}, q_{1}^{i+1 *}, \ldots, q_{1}^{\ell *}, 0, \ldots, 0\right)$ is more profitable than

$\left(q_{1}^{1 *}, \ldots, q_{1}^{i-1 *}, 0, q_{1}^{i+1 *}, \ldots, q_{1}^{\ell *}, 0, \ldots, 0\right)$ for any firm $i \in\{1, \ldots, \ell\}$.

That is (b) determines a condition under which at least $\ell$ firms store.

(c) No firm not storing in first period finds profitable to increase inventories to join the group of firms storing:

$\left(q_{1}^{1 *}, \ldots, q_{1}^{\ell *}, 0, \ldots, 0\right)$ is more profitable than $\left(q_{1}^{1 *}, \ldots, q_{1}^{\ell *}, 0, \ldots, 0, q_{1}^{i}, 0, \ldots, 0\right)$ for any $q_{1}^{i}$ such that $q_{1} \in B(\ell+1)$ and for any firm $i \in\{\ell+1, \ldots, n\}$.

That is (c) determines a condition under which at most $\ell$ firms store.

Let us start with case (a).

(a) The profit of firms storing is given by $\pi^{i}\left(q_{1}^{i}, q_{1}^{-i}\right)=\left(a-\frac{(n-\ell)(a-c)+\sum_{i=1}^{\ell} q_{1}^{i}}{n-\ell+1}\right) q_{1}^{i}-$ $(1+\rho) c q_{1}^{i}$. Its maximization with respect to inventories $q_{1}^{i}$ leads to the following first order conditions (FOCs) for firms $1, \ldots, \ell$ :

$$
a-\frac{(n-\ell)(a-c)}{n-\ell+1}-\frac{\sum_{i=1}^{\ell} q_{1}^{i}}{n-\ell+1}-\frac{q_{1}^{i}}{n-\ell+1}-(1+\rho) c=0 \text { for } i=1, \ldots, \ell .
$$

If an equilibrium exists it must satisfy all these FOCs. The candidate equilibrium for all firms storing $i=1, \ldots, \ell$, is therefore:

$$
q_{1}^{*}(\ell)=\frac{a-(n-\ell+1)(1+\rho) c+(n-\ell) c}{\ell+1}=\frac{a-c-(n-\ell+1) \rho c}{\ell+1} .
$$


Then we must check that the n-tuple obtained by satisfying all FOCs simultaneously is indeed "interior" to the region $B(\ell)$, that is $\left(q_{1}^{1 *}, \ldots, q_{1}^{\ell *}, 0, \ldots, 0\right)$ satisfies the conditions (29) and (30). Using the expression of $q_{1}^{*}(\ell)$, the first one simplifies into a condition on the unit cost of producing and storing the product in period $1,(1+\rho) c$ :

$$
\begin{aligned}
& q_{1}^{*}(\ell) \geq \frac{a-c-(\ell-1) q_{1}^{*}(\ell)}{n-\ell+2} \Leftrightarrow q_{1}^{*}(\ell) \geq \frac{a-c}{n+1} \Leftrightarrow \frac{a-c-(n-\ell+1) \rho c}{\ell+1} \geq \frac{a-c}{n+1} \\
\Leftrightarrow & \rho c \leq \frac{(n-\ell)(a-c)}{(n+1)(n-\ell+1)} .
\end{aligned}
$$

Similarly the second condition rewrites:

$$
\begin{aligned}
& 0 \leq \frac{a-c-\ell q_{1}^{*}(\ell)}{n-\ell+1} \Leftrightarrow q_{1}^{*}(\ell) \leq \frac{a-c}{\ell} \Leftrightarrow(\ell+1)(a-c) \geq \\
& \ell a-\ell(n-\ell+1)(1+\rho) c+\ell(n-\ell) c \Leftrightarrow \ell(n-\ell+1) \rho c \geq-a+c
\end{aligned}
$$

which always hold under (A.1). Consequently to summarize case (a), the ntuple $\left(q_{1}^{*}(\ell), \ldots, q_{1}^{*}(\ell), 0, \ldots, 0\right)$ with $\ell$ firms storing is in $B(\ell)$ if $(38)$ holds, that is if the cost of storage is small enough.

(b) As all firms earn the same payoff, the profit function of any firm storing $q_{1}^{*}(\ell)$ is equal to $\pi^{*}(\ell)=\left(a-S^{*}(\ell)-(1+\rho) c\right) q_{1}^{*}(\ell)$. Let us check it is not profitable for firm $\ell$ to store nothing and wait for the second period to produce, while firms $1, \ldots, \ell-1$ store $q_{1}^{*}(\ell)$.

We first need to determine to which subgame the deviation of firm $\ell$ leads to, that is to which subgame the n-tuple of inventories $\left(q_{1}^{*}(\ell), \ldots, q_{1}^{*}(\ell), 0, \ldots, 0\right)$ with only $\ell-1$ firms storing leads to, that is the equilibrium sales of the oligopolists. The conditions for firms 1 to $\ell-1$ to sell exactly their inventories while firms $\ell$ to $n$ store nothing (and wait for the second period to produce) are obtained by replacing $\ell$ with $\ell-1$ in (29) and (30):

$$
\begin{aligned}
& q_{1}^{\ell-1} \geq \frac{a-c-\sum_{i=1}^{\ell-2} q_{1}^{i}}{n-\ell+3} \\
& q_{1}^{\ell} \leq \frac{a-c-\sum_{i=1}^{\ell-1} q_{1}^{i}}{n-\ell+2} .
\end{aligned}
$$

Replacing each $q_{1}^{i}$ by $q_{1}^{*}(\ell)$ for $i=1, \ldots, \ell-1$ and $q_{1}^{\ell}$ by 0 in these inequalities gives a condition on the level $q_{1}^{*}(\ell)$ of individual inventories kept by firms $1, \ldots, \ell-1$ :

$$
q_{1}^{*}(\ell) \geq \frac{a-c-(\ell-2) q_{1}^{*}(\ell)}{n-\ell+3} \Leftrightarrow q_{1}^{*}(\ell) \geq \frac{a-c}{n+1}
$$


and

$$
0 \leq \frac{a-c-(\ell-1) q_{1}^{*}(\ell)}{n-\ell+2} \Leftrightarrow q_{1}^{*}(\ell) \leq \frac{a-c}{\ell-1} .
$$

We know from the first line in (38) and (39) that to be such that $q_{1}^{*}(\ell) \in B(\ell)$, $q_{1}^{*}(\ell)$ must verify $q_{1}^{*}(\ell) \geq(a-c) /(n+1)$ and $q_{1}^{*}(\ell) \leq(a-c) / \ell$. Respecting these two conditions imply that (42) and (43) also hold. Consequently the deviation of firm $\ell$ to a zero level of inventories when firms $1, \ldots, \ell-1$ store each $q_{1}^{*}(\ell)$ is such that the n-tuple $\left(q_{1}^{*}(\ell), \ldots, q_{1}^{*}(\ell), 0, \ldots, 0\right)$ with $\ell-1$ firms storing belongs to $B(\ell-1)$.

Substituting $\ell$ for $\ell-1$ in (27), firms $\ell, \ldots, n$ produce in second period a quantity given by

$$
s^{*}(\ell-1)=\frac{a-c-(\ell-1) q_{1}^{*}(\ell)}{n-\ell+2},
$$

and the aggregate quantity sold is equal to:

$$
S^{*}(\ell-1)=\frac{(n-\ell+1)(a-c)+(\ell-1) q_{1}^{*}(\ell)}{n-\ell+2} .
$$

Consequently we can compute and compare the profits of firm $\ell$ with and without deviating. Firm $\ell$ 's profit when storing $q_{1}^{*}(\ell)$ is larger than the profit it can obtain from not storing and waiting to the second period to produce and sell $s^{*}(\ell-1)$ when all other firms $1, \ldots, \ell-1$ store $q_{1}^{*}(\ell)$ if

$$
\begin{gathered}
\pi^{*}(\ell) \equiv\left(a-S^{*}(\ell)-(1+\rho) c\right) q_{1}^{*}(\ell) \geq \\
\left(a-c-S^{*}(\ell-1)\right) s^{\ell *}(\ell-1) \equiv \pi_{d e v}^{\ell}
\end{gathered}
$$

Checking that this deviation is not profitable gives another set of conditions on $q_{1}^{*}(\ell)$ we need to compare with the conditions obtained in (a).

Let us start to rewrite the profit obtained by firm $\ell$ when it produces $q_{1}^{*}(\ell)$ in first period using (28):

$$
\begin{aligned}
\pi^{*}(\ell) & =\left(\frac{a+(n-\ell) c-\ell q_{1}^{*}(\ell)}{n-\ell+1}-(1+\rho) c\right) q_{1}^{*}(\ell)=\left(\frac{(\ell+1) q_{1}^{*}(\ell)-\ell q_{1}^{*}(\ell)}{n-\ell+1}\right) q_{1}^{*}(\ell) \\
& =\frac{1}{n-\ell+1}\left(\frac{a-c-(n-\ell+1) \rho c}{\ell+1}\right)^{2}
\end{aligned}
$$

Remark that $(a-c-(n-\ell+1) \rho c)$ in the last expression is positive since $q_{1}^{*}(\ell)$ is positive under (39). 
Similarly the profit of firm $\ell$ when deviating to $q_{1}^{\ell}=0$ is equal to:

$$
\begin{aligned}
\pi_{d e v}^{\ell} & =\left(a-c-\frac{(n-\ell+1)(a-c)}{n-\ell+2}-\frac{(\ell-1) q_{1}^{*}(\ell)}{n-\ell+2}\right) \times \frac{a-c-(\ell-1) q_{1}^{*}(\ell)}{n-\ell+2} \\
& =\left(\frac{a-c-(\ell-1) q_{1}^{*}(\ell)}{n-\ell+2}\right)^{2}=\left(\frac{2(a-c)+(\ell-1)(n-\ell+1) \rho c}{(n-\ell+2)(\ell+1)}\right)^{2} .
\end{aligned}
$$

From its definition in the second line of the expression above, the profit $\pi_{d e v}^{\ell}$ is proportional to the square of $a-c-(\ell-1) q_{1}^{*}(\ell)$, which is positive under (39). Consequently the comparison between the two profits reduces to the comparison between the terms that are squared:

$$
\begin{aligned}
& \pi^{*}(\ell) \geq \pi_{d e v}^{\ell} \Leftrightarrow \frac{a-c-(n-\ell+1) \rho c}{(\ell+1) \sqrt{n-\ell+1}} \geq \frac{2(a-c)+(\ell-1)(n-\ell+1) \rho c}{(\ell+1)(n-\ell+2)} \\
\Leftrightarrow & (n-\ell+2)(a-c)-(n-\ell+2)(n-\ell+1) \rho c \geq \\
& 2 \sqrt{n-\ell+1}(a-c)+\sqrt{n-\ell+1}(\ell-1)(n-\ell+1) \rho c \\
\Leftrightarrow & (n-\ell+1)(\sqrt{n-\ell+1}(\ell-1)+n-\ell+2) \rho c \\
& \leq(n-\ell+2-2 \sqrt{n-\ell+1})(a-c)
\end{aligned}
$$

As the coefficient in factor of $\rho c$ is positive, this is equivalent to

$$
\rho c \leq \frac{(n-\ell+2-2 \sqrt{n-\ell+1})(a-c)}{(n-\ell+1)(\sqrt{n-\ell+1}(\ell-1)+n-\ell+2)} .
$$

To summarize case (b) if the cost of storage $\rho c$ verifies the condition above, the deviation from $q_{1}^{\ell}=q_{1}^{*}(\ell)$ to $q_{1}^{\ell}=0$ is not profitable for firm $\ell$; else it is profitable. Therefore respecting the condition (50) above ensures that at least $\ell$ firms are storing.

(c) We already proved that firm $n$ with the lowest level of inventories is always better off not storing at all. Consequently we do not need to check whether this firm finds profitable to store when firms $1, \ldots, n-1$ are storing, that is for $\ell=n-1$. In that case only the bounds we determined in (a) and (b) matter. However we need to check that storing is not profitable for firms $\ell+1, \ldots, n-1$ that do not store when $\ell \leq n-2$.

Since all firms who are selling more than their inventories in period 1 are not storing at all in period 1 , it suffices to check whether a profitable deviation exists for one of them, say $\ell+1$, to prove (c). First, this firm must choose $q_{1}^{\ell+1}$ 
such that the $(\ell+1)$-tuple $\left(q_{1}^{*}(\ell), \ldots, q_{1}^{*}(\ell), q_{1}^{\ell+1}, 0, \ldots, 0\right)$ belongs to $B(\ell+1)$, i.e. is such that firms 1 to $\ell+1$ sell their inventories and the others do not store. Indeed if it were not the case, firm $\ell+1$ would sell the same quantity than firms $\ell+2$ to $n$ while bearing the extra cost of carrying inventories, which of course would not be profitable. Second, the deviation $q_{1}^{\overline{1}+1}$ chosen must also be such that the profit $\pi_{d e v}^{\ell+1}\left(q_{1}^{\ell+1}\right)$ is maximal. We check these conditions in turn. Adapting again (29) and (30) to determine $B(\ell+1), q_{1}^{\ell+1}$ is sold in second period when firms $1, \ldots, \ell$ store each $q_{1}^{*}(\ell)$ if it verifies the two conditions:

$$
\begin{aligned}
& q_{1}^{\ell+1} \geq \frac{a-c-\ell q_{1}^{*}(\ell)}{n-\ell+1} \\
& 0 \leq \frac{a-c-\ell q_{1}^{*}(\ell)-q_{1}^{\ell+1}}{n-\ell} \Leftrightarrow q_{1}^{\ell+1} \leq a-c-\ell q_{1}^{*}(\ell) .
\end{aligned}
$$

The fact that $q_{1}^{*}(\ell)$ verifies $(39)$ insures that $q_{1}^{*}(\ell) \leq(a-c) / \ell$, and therefore $a-c-\overline{1} q_{1}^{*}(\ell) \geq 0$ so that the lower and the upper bounds on $q_{1}^{\ell+1}$ given in (51) are non-negative and such that $a-c-\ell q_{1}^{*}(\ell)>\frac{a-c-\ell q_{1}^{*}(\ell)}{n-\ell+1}$ for any $n \geq \ell+1$. Moreover since

$$
a-c-\ell q_{1}^{*}(\ell)=\frac{a-c+\ell(n-\ell+1) \rho c}{\ell+1}
$$

the RHS of the two inequalities (51) and (52) above define two bounds $U$ and $D$ functions of $(1+\rho) c$ such that:

$$
\begin{aligned}
& q_{1}^{\ell+1} \geq \frac{a-c+\ell(n-\ell+1) \rho c}{(\ell+1)(n-\ell+1)} \equiv D \\
& q_{1}^{\ell+1} \leq \frac{a-c+\ell(n-\ell+1) \rho c}{\ell+1} \equiv U .
\end{aligned}
$$

If these two conditions hold then aggregate sales are given by Proposition 1:

$$
S^{*}(\ell+1)=\frac{(n-\ell-1)(a-c)+\ell q_{1}^{*}(\ell)+q_{1}^{\ell+1}}{n-\ell}
$$

and the profit of firm $\ell+1$ is equal to:

$$
\pi^{\ell+1}=\left(a-\frac{(n-\ell-1)(a-c)+\ell q_{1}^{*}(\ell)+q_{1}^{\ell+1}}{n-\ell}-(1+\rho) c\right) q_{1}^{\ell+1} .
$$

This profit is maximal when $q_{1}^{\ell+1}$ solves the first order condition

$$
a-c-\frac{(n-\ell-1)(a-c)+\ell q_{1}^{*}(\ell)+q_{1}^{\ell+1}}{n-\ell}-\rho c-\frac{1}{n-\ell} q_{1}^{\ell+1}=0
$$


whose solution in $q_{1}^{\ell+1}$ is:

$$
\begin{aligned}
q_{\text {dev }}^{\ell+1}= & \frac{a-c-(n-\ell) \rho c-\ell q_{1}^{*}(\ell)}{2}=\frac{1}{2}\left(a-c-(n-\ell) \rho c-\ell \frac{a-c-(n-\ell+1) \rho c}{\ell+1}\right) \\
= & \frac{a-c-(\ell+1)(n-\ell) \rho c+\ell(n-\ell+1) \rho c}{2(\ell+1)}=\frac{a-c-(n-2 \ell) \rho c}{2(\ell+1)} .
\end{aligned}
$$

We have $q_{d e v}^{\ell+1} \geq U$ if and only if $\frac{a-c-(n-2 \ell) \rho c}{2(\ell+1)} \geq \frac{a-c+\ell(n-\ell+1) \rho c}{\ell+1}$ that is

$$
-(a-c) \geq(2 \ell(n-\ell)+n) \rho c
$$

which is impossible as the LHS of this inequality is negative while the RHS is positive. Consequently we always have $q_{d e v}^{\ell+1}<U$.

Similarly $q_{d e v}^{\ell+1} \leq D$ if and only if

$$
\begin{aligned}
& \frac{a-c-(n-2 \ell) \rho c}{2(\ell+1)} \leq \frac{a-c+\ell(n-\ell+1) \rho c}{(\ell+1)(n-\ell+1)} \\
\Leftrightarrow & (n-\ell+1)(a-c-(n-2 \ell) \rho c) \leq 2(a-c)+2 \ell(n-\ell+1) \rho c \\
\Leftrightarrow & (n-\ell-1)(a-c) \leq n(n-\ell+1) \rho c \Leftrightarrow \frac{n-\ell-1}{n(n-\ell+1)}(a-c) \leq \rho c .
\end{aligned}
$$

Consequently if $\rho c \geq \frac{n-\ell-1}{n(n-\ell+1)}(a-c)$ then by concavity of the profit (57) in $q_{1}^{\ell+1}$, the deviation to consider is $q_{d e v^{\prime \prime}}^{\ell+1}=D$ instead of $q_{d e v}^{\ell+1}$. This case is analyzed in (c.1) below. If on the other hand $\rho c \leq \frac{n-\ell-1}{n(n-\ell+1)}(a-c)$ then the deviation to consider is $q_{d e v}^{\ell+1}$. This case is analyzed in (c.2) below.

Sub-case (c.1): $\rho c \geq \frac{n-\ell-1}{n(n-\ell+1)}(a-c)$. In that case firm $\ell+1$ optimum in $B(\ell+1)$ is to choose $q_{d e v^{\prime}}^{\ell+1}=\frac{a-c-\ell q_{1}^{*}(\ell)}{n-\ell+1}$. As recalled in the introduction of this proof the quantity sold by firm $\ell+1$ when it does not store is given by $s^{(\ell+1) *}(\ell)=\left(a-c-\overline{1} q_{1}^{*}(\ell)\right) /(n-\ell+1)$, which is identical to $q_{d e v^{\prime}}^{\ell+1}$. Consequently aggregate sales $S^{*}(\ell+1)$ and $S^{*}(\ell)$ are identical to each other for the output choices of firm $\ell+1$ we consider. As production is more costly in first than in second period due to discounting, it is clearly not profitable for firm $\ell+1$ to deviate. Consequently for these values of $\rho c, \ell+1$ does not deviate from $q_{1}^{\ell+1 *}=0$. 
Sub-case (c.2): $\rho c \leq \frac{n-\ell-1}{n(n-\ell+1)}(a-c)$. In that case firm $\ell+1$ optimum in $B(\ell+1)$ is to choose $q_{d e v}^{\ell+1}$. This deviation is not profitable if the profit obtained by not storing exceeds the profit obtained by choosing $q_{d e v}^{\ell+1}$ above, that is:

$$
\begin{aligned}
& \pi_{*}^{\ell+1} \geq \pi_{\text {dev }}^{\ell+1} \\
\Leftrightarrow & \left(a-c-S^{*}(\ell)\right) s^{(\ell+1) *}(\ell) \geq\left(a-S^{*}(\ell+1)-(1+\rho) c\right) q_{d e v}^{\ell+1}
\end{aligned}
$$

where

$$
\begin{aligned}
& S^{*}(\ell+1)=\left((n-\ell-1)(a-c)+\ell q_{1}^{*}(\ell)+q_{d e v}^{\ell+1}\right) /(n-\ell) \\
& S^{*}(\ell)=\left((n-\ell)(a-c)+\ell q_{1}^{*}(\ell)\right) /(n-\ell+1) \\
& s^{(\ell+1) *}(\ell)=\left(a-c-\ell q_{1}^{*}(\ell)\right) /(n-\ell+1) .
\end{aligned}
$$

Substituting these terms in $\pi_{d e v}^{\ell+1}$ and using the fact that $q_{d e v}^{\ell+1}=\left(a-c-(n-\ell) \rho c-\ell q_{1}^{*}(\ell)\right) / 2$, it comes:

$$
\begin{aligned}
\pi_{d e v}^{\ell+1} & =\left(\frac{a-c-\ell q_{1}^{*}(\ell)-q_{d e v}^{\ell+1}-(n-\ell) \rho c}{n-\ell}\right) q_{d e v}^{\ell+1} \\
& =\frac{\left(a-c-(n-\ell) \rho c-\ell q_{1}^{*}(\ell)\right)^{2}}{4(n-\ell)}=\frac{(a-c-(n-2 \ell) \rho c)^{2}}{4(n-\ell)(\ell+1)^{2}} .
\end{aligned}
$$

Similarly when firm $\ell+1$ chooses not to store, the profit $\pi_{*}^{\ell+1}$ it obtains can be simplified, using 53 , into:

$$
\pi_{*}^{\ell+1}=\left(\frac{a-c-\ell q_{1}^{*}(\ell)}{n-\ell+1}\right)^{2}=\left(\frac{a-c+\ell(n-\ell+1) \rho c}{(n-\ell+1)(\ell+1)}\right)^{2} .
$$

Then since $q_{d e v}^{\ell+1}$ and $s^{(\ell+1) *}(\ell)$ are positive, the profit comparison is equivalent to compare the terms that are squared:

$$
\begin{gathered}
\pi_{*}^{\ell+1} \geq \pi_{d e v}^{\ell+1} \Leftrightarrow \frac{a-c+\ell(n-\ell+1) \rho c}{(n-\ell+1)(\ell+1)} \geq \frac{a-c-(n-2 \ell) \rho c}{2 \sqrt{n-\ell}(\ell+1)} \\
\Leftrightarrow(n-\ell+1)(2 \ell \sqrt{n-\ell}+n-2 \ell) \rho c \geq(n-\ell+1-2 \sqrt{n-\ell})(a-c)
\end{gathered}
$$

The coefficient in front of $(a-c)$ on the RHS of this inequality is always positive, and the coefficient $2 \ell \sqrt{n-\ell}+n-2 \ell$ in front of $\rho c$ on the LHS as well: indeed it defines a function of $n$ that is strictly increasing. Therefore $2 \ell \sqrt{n-\ell}+n-2 \ell$ is strictly larger than the value it takes for the smallest $n$ possible, $n=\ell+1$,

$$
2 \ell \sqrt{n-\ell}+n-2 \ell \geq 2 \ell \sqrt{\ell+1-\ell}+\ell+1-2 \ell=\ell+1 \geq 0 .
$$


Therefore

$$
\pi_{*}^{\ell+1} \geq \pi_{d e v}^{\ell+1} \Leftrightarrow \rho c \geq \frac{(n-\ell+1-2 \sqrt{n-\ell})(a-c)}{(n-\ell+1)(2 \ell \sqrt{n-\ell}+n-2 \ell)} .
$$

We can compare this bound with the bound of region (c.2) by simply comparing the terms in factor of $a-c$. It comes:

$$
\frac{n-\ell+1-2 \sqrt{n-\ell}}{(n-\ell+1)(2 \ell \sqrt{n-\ell}+n-2 \ell)} \leq \frac{n-\ell-1}{n(n-\ell+1)}
$$

if and only if

$$
n(n-\ell+1-2 \sqrt{n-\ell}) \leq(2 \ell \sqrt{n-\ell}+n-2 \ell)(n-\ell-1)
$$

Collecting the terms multiplied by $2 \sqrt{n-\ell}$ and separating them from the rest gives

$$
\begin{aligned}
& 0 \leq 2 \sqrt{n-\ell}(n+\ell(n-\ell-1))-n(n-\ell+1)+(n-2 \ell)(n-\ell-1) \\
\Leftrightarrow & 0 \leq 2 \sqrt{n-\ell}(n-\ell)(\ell+1)-n(n-\ell)-n+n(n-\ell)-n-2 \ell(n-\ell)+2 \ell \\
\Leftrightarrow & 0 \leq 2 \sqrt{n-\ell}(n-\ell)(\ell+1)-2(n-\ell)(\ell+1) \\
\Leftrightarrow & 0 \leq 2(\sqrt{n-\ell}-1)(n-\ell)(\ell+1) .
\end{aligned}
$$

Remark that $\sqrt{n-\ell}-1 \geq 0$. Therefore the comparison between this bound and the bound of region (c.2) ensures that firm $\ell+1$ does not find profitable to deviate from $q_{1}^{\ell+1}=0$ if $(1+\rho) c$ satisfies

$$
\frac{(n-\ell+1-2 \sqrt{n-\ell})(a-c)}{(n-\ell+1)(2 \ell \sqrt{n-\ell}+n-2 \ell)} \leq \rho c \leq \frac{(n-\ell-1)(a-c)}{n(n-\ell+1)}
$$

else it does.

To summarize case (c), firm $\ell+1$ does not find profitable to deviate from $q_{1}^{\ell+1}=0$ to $q_{d e v}^{\ell+1}$ if

$$
\rho c \geq \frac{n-\ell+1-2 \sqrt{n-\ell}}{(n-\ell+1)(2 \ell \sqrt{n-\ell}+n-2 \ell)}(a-c),
$$

while if

$$
\rho c \leq \frac{n-\ell+1-2 \sqrt{n-\ell}}{(n-\ell+1)(2 \ell \sqrt{n-\ell}+n-2 \ell)}(a-c)
$$

it does. Respecting the condition (75) above ensures that at most $\ell$ firms store. 
Step 3. Non-emptiness of the set of parameters such that an equilibrium with $\ell$ firms storing exists.

To start with, remark that the bound on $\rho c$ we obtained in (50) of step (b) is lower than the bound we obtained in (38) of step (a). The comparison is equivalent to verify that

$$
\begin{aligned}
& \frac{n-\ell+2-2 \sqrt{n-\ell+1}}{(n-\ell+1)(\sqrt{n-\ell+1}(\ell-1)+n-\ell+2)} \leq \frac{n-\ell}{(n+1)(n-\ell+1)} \\
\Leftrightarrow & (\sqrt{n-\ell+1}(\ell-1)+n-\ell+2)(n-\ell) \geq(n+1)(n-\ell+2-2 \sqrt{n-\ell+1}) \\
\Leftrightarrow & -(\ell+1)(n-\ell+2)+((\ell-1)(n-\ell)+2(n+1)) \sqrt{n-\ell+1} \geq 0 .
\end{aligned}
$$

Remark that $(\ell-1)(n-\ell)+2(n+1)=n+n \ell-\ell^{2}+\ell+2=(\ell+1)(n-\ell+2)$; then the inequality above is equivalent to $(\ell+1)(n-\ell+2)(\sqrt{n-\ell+1}-1) \geq 0$ which always hold. Then we need to compare the bound obtained in (50) with the bound determined by (75). This is again equivalent to compare the terms in factor of $(a-c)$. We have

$$
\begin{aligned}
& \frac{n-\ell+2-2 \sqrt{n-\ell+1}}{(n-\ell+1)(\sqrt{n-\ell+1}(\ell-1)+n-\ell+2)} \geq \frac{n-\ell+1-2 \sqrt{n-\ell}}{(n-\ell+1)(2 \ell \sqrt{n-\ell}+n-2 \ell)} \\
\Leftrightarrow & (2 \ell \sqrt{n-\ell}+n-2 \ell)(n-\ell+2-2 \sqrt{n-\ell+1}) \geq \\
& (\sqrt{n-\ell+1}(\ell-1)+n-\ell+2)(n-\ell+1-2 \sqrt{n-\ell}) \\
\Leftrightarrow & (n-\ell+2)(-\ell-1)+(n-\ell+2)(\ell+1) 2 \sqrt{n-\ell} \\
& +(-2(n-2 \ell)-(\ell-1)(n-\ell+1)) \sqrt{n-\ell+1} \\
& +(-4 \ell+2(\ell-1)) \sqrt{n-\ell} \sqrt{n-\ell+1} \geq 0 \\
\Leftrightarrow & (n-\ell+2)(\ell+1)(2 \sqrt{n-\ell}-1) \\
& +\left(-n+2 \ell-n \ell+\ell^{2}+1\right) \sqrt{n-\ell+1}-2(\ell+1) \sqrt{n-\ell} \sqrt{n-\ell+1} \geq 0
\end{aligned}
$$

Remark that $-n+2 \ell-n \ell+\ell^{2}+1=(\ell+1)(-n+\ell+1)$. The inequality above can be simplified by dividing both sides by $\ell+1$, giving

$$
(n-\ell+2)(2 \sqrt{n-\ell}-1)-(n-\ell-1) \sqrt{n-\ell+1}-2 \sqrt{n-\ell} \sqrt{n-\ell+1} \geq 0 .
$$

The LHS of the inequality above defines a function of $n-\ell$ that is strictly increasing. 
Indeed the derivative of the LHS with respect to $n-\ell$ is equal to

$$
2 \sqrt{n-\ell}-1+\frac{n-\ell+2}{\sqrt{n-\ell}}-\frac{n-\ell-1+2 \sqrt{n-\ell}}{2 \sqrt{n-\ell}}-\sqrt{n-\ell+1}\left(1+\frac{1}{\sqrt{n-\ell}}\right)
$$

which is positive after reducing to the same denominator $2 \sqrt{(n-\ell)(n-\ell+1)}$ and verifying the sign of the numerator. Indeed let us change temporarily the notation, introducing $x=n-\ell$. Then it rewrites:

$$
2 \sqrt{x}-1+\frac{x+2}{\sqrt{x}}-\frac{x-1+2 \sqrt{x}}{2 \sqrt{x+1}}-\sqrt{x+1}\left(1+\frac{1}{\sqrt{x}}\right)
$$

which is, by multiplying by $2 \sqrt{x(x+1)}$, of the same sign than:

$$
\begin{aligned}
& (2 \sqrt{x}-1) 2 \sqrt{x(x+1)}+(x+2) 2 \sqrt{x+1}-(x-1+2 \sqrt{x}) \sqrt{x}-2 \sqrt{x}(x+1)\left(1+\frac{1}{\sqrt{x}}\right) \\
= & 4 x \sqrt{x+1}-2 \sqrt{x(x+1)}+2(x+2) \sqrt{x+1}-(x-1) \sqrt{x}-2 x-2(x+1) \sqrt{x}-2(x+1) \\
= & (6 x+4) \sqrt{x+1}-2 \sqrt{x(x+1)}-(3 x+1) \sqrt{x}-4 x-2 \\
= & (6 x+4-2 \sqrt{x}) \sqrt{x+1}-(4 x+2+(3 x+1) \sqrt{x}) .
\end{aligned}
$$

Then

$$
\begin{aligned}
& (6 x+4-2 \sqrt{x}) \sqrt{x+1}-(4 x+2+(3 x+1) \sqrt{x}) \geq 0 \\
\Leftrightarrow & (6 x+4-2 \sqrt{x})^{2}(x+1) \geq(4 x+2+(3 x+1) \sqrt{x})^{2} \\
\Leftrightarrow & (x+1)(6 x+4)^{2}-4(6 x+4)(x+1) \sqrt{x}+4 x(x+1) \geq \\
& (4 x+2)^{2}+2(4 x+2)(3 x+1) \sqrt{x}+x(3 x+1)^{2} \\
\Leftrightarrow & (x+1)(6 x+4)^{2}+4 x(x+1)-(4 x+2)^{2}-x(3 x+1)^{2} \geq \\
& 4(6 x+4)(x+1) \sqrt{x}-2(4 x+2)(3 x+1) \sqrt{x} \\
\Leftrightarrow & x(6 x+4-3 x-1)(6 x+4+3 x+1)+(6 x+4-4 x-2)(6 x+4+4 x+2)+4 x(x+1) \geq \\
& \left(24 x^{2}+40 x+16-24 x^{2}-20 x-4\right) \sqrt{x} \\
\Leftrightarrow & x(3 x+3)(9 x+5)+(2 x+2)(10 x+6)+4 x(x+1) \geq(20 x+12) \sqrt{x} \\
\Leftrightarrow & (x+1)(3 x(9 x+5)+20 x+12+4 x) \geq(20 x+12) \sqrt{x} \\
\Leftrightarrow & (x+1)\left(27 x^{2}+39 x+12\right) \geq(20 x+12) \sqrt{x} \\
\Leftrightarrow & (x+1)^{2}(27 x+12) \geq(20 x+12) \sqrt{x}
\end{aligned}
$$

which holds for any $x \geq 1$. Therefore the LHS of the inequality (79) defines a function of $n-\ell$ that is strictly increasing. Then as $n-\ell \geq 1$, it suffices to check 
that

$$
(n-\ell+2)(2 \sqrt{n-\ell}-1)-(n-\ell-1) \sqrt{n-\ell+1}-2 \sqrt{n-\ell} \sqrt{n-\ell+1} \geq 0
$$

for $n-\ell=1$. It simplifies into $3-2 \sqrt{2} \geq 0$ which always holds. To conclude this part of proof the $n$-tuple $\left(q_{1}^{1 *}, \ldots, q_{1}^{\ell *}, q_{1}^{\ell+1 *}, \ldots, q_{1}^{n *}\right)=\left(q_{1}^{*}(\ell), \ldots, q_{1}^{*}(\ell), 0, \ldots, 0\right)$ is an equilibrium in pure strategies of the game if and only if

$$
\frac{(n-\ell+2-2 \sqrt{n-\ell+1})(a-c)}{(n-\ell+1)(\sqrt{n-\ell+1}(\ell-1)+n-\ell+2)} \geq \rho c \geq \frac{(n-\ell+1-2 \sqrt{n-\ell})(a-c)}{(n-\ell+1)(2 \ell \sqrt{n-\ell}+n-2 \ell)} .
$$

Step 4: all possible values of the model parameters are covered.

It remains to prove that we have covered all possible values of the model parameters. We established in steps 1 and 2 conditions on $\rho$ such that an equilibrium exists, for any values of $a$ and $c$ satisfying (A.1). We can now check that these conditions cover all possible values of $\rho$ for $a$ and $c$ satisfying (A.1). Let us rewrite the second condition as:

$$
\begin{aligned}
& \frac{\rho c}{a-c} \geq \frac{(n-\ell+1-2 \sqrt{n-\ell})}{(n-\ell+1)(2 \ell \sqrt{n-\ell}+n-2 \ell)} \equiv L(n, \ell) \\
& \frac{\rho c}{a-c} \leq \frac{(n-\ell+2-2 \sqrt{n-\ell+1})}{(n-\ell+1)(\sqrt{n-\ell+1}(\ell-1)+n-\ell+2)} \equiv U(n, \ell)
\end{aligned}
$$

First remark that $U(n, 1)=\frac{n+1-2 \sqrt{n}}{n(n+1)}$. This is the bound we determined in for the Cournot-Nash to be an equilibrium. Consequently for $\frac{\rho c}{a-c} \geq U(n, 1)$, the CournotNash is an equilibrium of the game. Second, we prove now that $U(n, \ell+1) \geq L(n, \ell)$ for any $\ell=1, \ldots, n-2$, meaning that the game may have several equilibria for the same set of parameters. Indeed, bearing in mind that the numerators of $L(n, \ell)$ and $U(n, \ell)$ are positive for any $\ell$,

$$
\begin{aligned}
& U(n, \ell+1) \geq L(n, \ell) \\
\Leftrightarrow & \frac{(n-\ell+1-2 \sqrt{n-\ell})}{(n-\ell)(\ell \sqrt{n-\ell}+n-\ell+1)} \geq \frac{(n-\ell+1-2 \sqrt{n-\ell})}{(n-\ell+1)(2 \ell \sqrt{n-\ell}+n-2 \ell)} \\
\Leftrightarrow & (n-\ell)(\ell \sqrt{n-\ell}+n-\ell+1) \leq(n-\ell+1)(2 \ell \sqrt{n-\ell}+n-2 \ell) \\
\Leftrightarrow & (2(n-\ell+1)-(n-\ell)) \ell \sqrt{n-\ell}-(n-\ell+1) \ell \geq 0 \\
\Leftrightarrow & (n-\ell+2) \ell \sqrt{n-\ell}-(n-\ell+1) \ell \geq 0 \\
\Leftrightarrow & (n-\ell+2) \sqrt{n-\ell}-(n-\ell+1) \geq 0
\end{aligned}
$$


which always hold. Finally, the condition we established for $\ell=n-1$ is exactly $\frac{\rho c}{a-c} \leq L(n, n-2)$. Consequently we have covered all possible values of $\rho$, and the game always possesses an equilibrium in pure strategies. 
Table 1: Computations of the equilibria and comparison with Cournot-Nash: Example.

\begin{tabular}{|c|c|c|c|c|c|c|c|c|c|c|c|c|}
\hline$\rho=0.03$ & $a=2.5$ & $c=1$ & & & & & & & & & & \\
\hline $\begin{array}{l}n \\
(1)\end{array}$ & $\begin{array}{l}\ell \text { min. } \\
(2)\end{array}$ & $\begin{array}{l}\ell \max . \\
(3)\end{array}$ & $\begin{array}{c}\ell \times q_{1}^{i *} \text { at }(2) \\
(4)\end{array}$ & $\begin{array}{c}\ell \times q_{1}^{i *} \text { at }(3) \\
(5)\end{array}$ & $\begin{array}{c}(n-\ell) \times s^{i *} \text { at }(2) \\
(6)\end{array}$ & $\begin{array}{c}(n-\ell) \times s^{i *} \text { at }(3) \\
(7)\end{array}$ & $\begin{array}{c}S^{*} \text { at }(2) \\
(8)\end{array}$ & $\begin{array}{c}S^{*} \text { at }(3) \\
(9)\end{array}$ & $\begin{array}{c}S^{*} \text { at Cournot } \\
(10)\end{array}$ & $\begin{array}{l}\text { HHI at (2) } \\
\text { (11) }\end{array}$ & $\begin{array}{l}\text { HHI at (3) } \\
\text { (12) }\end{array}$ & $\begin{array}{l}\text { HHI at (10) } \\
\text { (13) }\end{array}$ \\
\hline 2 & - & 1 & - & 0.720 & - & 0.390 & - & 1.110 & 1.000 & - & 5441.928 & 5000.000 \\
\hline 4 & - & 2 & - & 0.940 & - & 0.373 & - & 1.313 & 1.200 & - & 2965.420 & 2500.000 \\
\hline 5 & 2 & 3 & 0.920 & 1.058 & 0.435 & 0.295 & 1.355 & 1.353 & 1.250 & 2648.521 & 2275.686 & 2000.000 \\
\hline 6 & 2 & 3 & 0.900 & 1.035 & 0.480 & 0.349 & 1.380 & 1.384 & 1.286 & 2429.112 & 2076.586 & 1666.667 \\
\hline 7 & 3 & 4 & 1.013 & 1.104 & 0.390 & 0.297 & 1.403 & 1.401 & 1.313 & 1930.567 & 1702.195 & 1428.571 \\
\hline 8 & 3 & 4 & 0.990 & 1.080 & 0.425 & 0.336 & 1.415 & 1.416 & 1.333 & 1812.109 & 1595.088 & 1250.000 \\
\hline 10 & 3 & 5 & 0.945 & 1.100 & 0.486 & 0.333 & 1.431 & 1.433 & 1.364 & 1619.032 & 1286.101 & 1000.000 \\
\hline 11 & 3 & 5 & 0.923 & 1.075 & 0.513 & 0.364 & 1.436 & 1.439 & 1.375 & 1535.725 & 1222.482 & 909.091 \\
\hline 12 & 3 & 5 & 0.900 & 1.050 & 0.540 & 0.394 & 1.440 & 1.444 & 1.385 & 1458.333 & 1164.109 & 833.333 \\
\hline 13 & 3 & 5 & 0.878 & 1.025 & 0.566 & 0.422 & 1.443 & 1.447 & 1.393 & 1385.667 & 1109.641 & 769.231 \\
\hline 14 & 2 & 4 & 0.740 & 0.936 & 0.702 & 0.513 & 1.442 & 1.449 & 1.400 & 1514.957 & 1168.819 & 714.286 \\
\hline 15 & 2 & 4 & 0.720 & 0.912 & 0.724 & 0.539 & 1.444 & 1.451 & 1.406 & 1436.043 & 1113.075 & 666.667 \\
\hline 16 & 2 & 4 & 0.700 & 0.888 & 0.747 & 0.565 & 1.447 & 1.453 & 1.412 & 1360.934 & 1059.841 & 625.000 \\
\hline 17 & 2 & 4 & 0.680 & 0.864 & 0.769 & 0.591 & 1.449 & 1.455 & 1.417 & 1289.254 & 1008.862 & 588.235 \\
\hline 18 & 2 & 3 & 0.660 & 0.765 & 0.791 & 0.689 & 1.451 & 1.454 & 1.421 & 1220.719 & 1072.360 & 555.556 \\
\hline 19 & 2 & 3 & 0.640 & 0.743 & 0.812 & 0.713 & 1.452 & 1.455 & 1.425 & 1155.107 & 1017.494 & 526.316 \\
\hline 21 & 2 & 3 & 0.600 & 0.698 & 0.855 & 0.760 & 1.455 & 1.458 & 1.432 & 1031.991 & 914.227 & 476.190 \\
\hline 22 & 2 & 3 & 0.580 & 0.675 & 0.876 & 0.784 & 1.456 & 1.459 & 1.435 & 974.234 & 865.643 & 454.545 \\
\hline 23 & 1 & 2 & 0.405 & 0.560 & 1.047 & 0.897 & 1.452 & 1.457 & 1.438 & 1013.965 & 918.883 & 434.783 \\
\hline 24 & 1 & 2 & 0.390 & 0.540 & 1.064 & 0.918 & 1.454 & 1.458 & 1.440 & 952.491 & 865.861 & 416.667 \\
\hline 25 & 1 & 2 & 0.375 & 0.520 & 1.080 & 0.939 & 1.455 & 1.459 & 1.442 & 893.825 & 815.104 & 400.000 \\
\hline 26 & 1 & 2 & 0.360 & 0.500 & 1.096 & 0.960 & 1.456 & 1.460 & 1.444 & 837.877 & 766.560 & 384.615 \\
\hline 27 & 1 & 2 & 0.345 & 0.480 & 1.112 & 0.981 & 1.457 & 1.461 & 1.446 & 784.571 & 720.184 & 370.370 \\
\hline 28 & - & 1 & - & 0.330 & - & 1.128 & - & 1.458 & 1.448 & - & 733.841 & 357.143 \\
\hline 29 & - & 1 & - & 0.315 & - & 1.144 & - & 1.459 & 1.450 & - & 685.632 & 344.828 \\
\hline 30 & - & 1 & - & 0.300 & - & 1.160 & - & 1.460 & 1.452 & - & 639.895 & 333.333 \\
\hline 31 & - & 1 & - & 0.285 & - & 1.176 & - & 1.461 & 1.453 & - & 596.587 & 322.581 \\
\hline 32 & - & 1 & - & 0.270 & - & 1.192 & - & 1.462 & 1.455 & - & 555.672 & 312.500 \\
\hline 33 & - & 1 & - & 0.255 & - & 1.207 & - & 1.462 & 1.456 & - & 517.117 & 303.030 \\
\hline 34 & - & 0 & - & 0.000 & - & 1.457 & - & 1.457 & 1.457 & - & 294.118 & 294.118 \\
\hline$+\infty$ & - & 0 & - & 0 & - & 1.5 & - & 1.5 & 1.5 & - & 0 & 0 \\
\hline
\end{tabular}

